\title{
Topology of generalized spinors and chiral anomaly
}

\author{
Ho-Ung Yee ${ }^{1,2}$ and Piljin Yi® ${ }^{3}$ \\ ${ }^{1}$ Department of Physics, University of Illinois at Chicago, Chicago, Illinois 60607, USA \\ ${ }^{2}$ Kadanoff Center for Theoretical Physics, University of Chicago, Chicago, Illinois 60637, USA \\ ${ }^{3}$ School of Physics, Korea Institute for Advanced Study, Seoul 02455, Korea
}

(Received 20 October 2019; accepted 23 January 2020; published 10 February 2020)

\begin{abstract}
Weyl fermions with nonlinear dispersion have appeared in real-world systems, such as in the Weyl semimetals and topological insulators. We consider the most general form of Dirac operators and study its topological properties embedded in the chiral anomaly, in the index theorem, and in the odd-dimensional partition function, by employing the heat kernel. We find that all of these topological quantities are enhanced by a winding number defined by the Dirac operator in the momentum space, regardless of the spacetime dimensions. The chiral anomaly in $d=3+1$, in particular, is also confirmed via the conventional Feynman diagram. These interconnected results allow us to clarify the relationship between the chiral anomaly and the Chern number of the Berry connection, under dispute in some recent literature, and also lead to a compact proof of the Nielsen-Ninomiya theorem.
\end{abstract}

DOI: 10.1103/PhysRevD.101.045007

\section{INTRODUCTION}

In quantum field theories with fermions, we often encounter topological properties, with the chiral anomaly being perhaps the best-known such effect. Although initially derived from Feynman diagrams [1,2], its topological nature became quickly apparent via Fujikawa's alternative explanation [3] as the failure of the measure to be invariant under chiral rotations, which in turn translates to the Atiyah-Singer index density of the Dirac operator.

In recent years, chiral anomalies and other topological aspects emerged as relevant and useful concepts in condensed matter systems as well, notably in Weyl semimetals and topological insulators [4-6]. In the study of such systems, one encounters fermion systems of a more general kind than those familiar to high-energy physics. Instead of the usual Dirac operator, linear in the spacetime derivatives, a modified Hamiltonian of type

$$
H \sim \sigma^{+}\left(-i D_{+}\right)^{n}+\sigma^{-}\left(-i D_{-}\right)^{n}+\sigma^{3}\left(-i D_{3}\right)
$$

on a flat spatial $\mathbb{R}^{3}$ has appeared in the context of the Weyl semimetal. A chiral two-component fermion with such a generalized Dirac operator as the Hamiltonian is expected to suffer $n$ times the usual chiral anomaly. This was initially motivated by the merging of a pair of chiral Dirac cones in

Published by the American Physical Society under the terms of the Creative Commons Attribution 4.0 International license. Further distribution of this work must maintain attribution to the author(s) and the published article's title, journal citation, and DOI. Funded by SCOAP. the Brillouin zone, while more direct demonstrations via the Fujikawa method were recently given for $n=2$ and $n=3[7,8]$. Although this Hamiltonian is natural from the coalescence of several Weyl cones in the Brillouin zone, its topological equivalence to multiple Weyl fermions, as manifest in the anomaly, is hardly immediate from the usual continuum field theory viewpoint.

This begs for general inquiries into the anomaly and other topological aspects for fermions whose spacetime Dirac operator takes the most general form

$$
\gamma^{\mu} \mathcal{P}_{\mu}(-i D)
$$

where $-i D_{\mu}$ is the covariant momentum operator and $\mathcal{P}_{\mu}$ denotes polynomials, or even arbitrary smooth functions thereof. In the end, we will compute the index density, the anomaly, and also the anomalous phase of the partition function in odd spacetime dimensions, and find that all of these are minimally modified by the winding number of the map $K_{\mu} \rightarrow \mathcal{P}_{\mu}(K)$. Apart from this overall factor, the structure of the anomaly and the phase of the partition function remain intact. The same computation can be manipulated to show that this winding number is alternatively computed by counting the critical points $K_{*}$, defined by $\mathcal{P}\left(K_{*}\right)=0$, weighted by parities.

The latter should be reminiscent of the Morse theory [9] for those who are familiar with index theorems, but at the same time, this alternative picture shows how the winding number information of the generalized Dirac operator is connected to the Dirac/Weyl cones in the momentum space, in a way that has been fruitfully used in the condensed matter literature. In particular, the two 
alternative interpretations via the winding number and the Morse counting represent, respectively, the ultraviolet and the infrared viewpoints of one and the same quantity. The former viewpoint will connect to topological objects known in the momentum space as the Berry monopole, whose quantized flux can be also related to the $d=2+1$ topological insulator in the condensed matter literature [10-16].

Extending the discussion to odd spacetime dimensions, one finds a similar modification of the anomalous phase of the partition function. Given an odd-dimensional Dirac operator, this phase is computed by the eta invariant, which in turn is related to the Chern-Simons action. We will also see how this Chern-Simons effective action is also multiplicatively enhanced by the same kind of winding number as in even dimensions. If we consider this odd-dimensional spacetime as a flat boundary of an even-dimensional halfspacetime, an Atiyah-Patodi-Singer index theorem holds, again with the new overall multiplicative factor by the same winding number. This also means that the connection via the APS index theorem [17] between the $d=2+1$ boundary fermions and a bulk $d=3+1$ topological field theory carries over verbatim: much as in even-dimensional anomalies, the odd-dimensional anomalous phase does not distinguish between $N$ ordinary Dirac fermions and a generalized Dirac fermion with the winding number $N$.

It is our aim to derive these general results, and to explore their physical consequences. The starting point of this investigation is the chiral anomaly for such generalized Weyl fermions, which is one of the most robust handles we have on all quantum fermions with continuous classical symmetries. The chiral anomaly, many decades after its initial discovery, can be still mysterious. On the one hand, it is an infrared phenomenon of anomalous particle creation and annihilation at zero energy (level crossing point), in background field configurations where both parity and time-reversal symmetries are broken. On the other hand, its topological nature makes it computable also in ultraviolet scales, leading to its expression in terms of the local topological density of background fields. This infraredultraviolet connection is a profound characteristic of the chiral anomaly, which, when formulated in Euclidean space, leads to its deep connection to the index theorems in mathematics.

The infrared-ultraviolet connection of the chiral anomaly may also manifest itself in momentum space. In the infrared viewpoint, the anomaly should be given by contributions from local level-crossing points, where in- and outflows of particle numbers happen. Since the particle number is conserved away from these points due to the Liouville theorem [18], the same anomaly may also be seen in the ultraviolet region of large momenta, captured by some topology of the theory in consideration.

Can we prove the existence of such an infraredultraviolet connection in momentum space? If yes, what topology of the fermion theory in large momenta contains the information of the infrared chiral anomaly? One of our main results in this work is to provide a rigorous answer to this question. We show the existence of an infraredultraviolet connection of the chiral anomaly in momentum space for a general class of theories, where the Dirac operator is an arbitrary polynomial of covariant derivatives. In particular, we prove in Sec. III A that the topology of the Berry curvature of a projected chiral spinor in the asymptotically large momentum region carries precisely the same information of chiral anomaly in the infrared.

The Berry curvature of chiral spinors is an essential ingredient of the kinetic description of chiral particles in phase space, the chiral kinetic theory [19-23], where semiclassical approximation is justified at large momenta. It is responsible for many novel transport phenomena in the real-time dynamics of (pseudo)chiral fermion systems, in both the condensed matter physics of Dirac/Weyl semimetals [19,24-29] and the physics of quark-gluon plasma in relativistic heavy-ion collisions [30,31]. This includes most notably the chiral magnetic effect [32-34], the chiral vortical effect [35,36], and the anomalous Hall effect [37-40]. ${ }^{1}$ Within the kinetic theory description, it has been argued that the chiral anomaly may also be explained by the same Berry curvature $[19,20,46]$.

The kinetic theory description as well as the concept of the Berry phase breaks down for some states-e.g., those near level-crossing points-while the anomaly should be universal regardless of particular states or approximations [47-49]. As such, the exact nature of these relations needs further clarification. Nevertheless, the appearance of the common topological quantity-namely, the winding number of the Dirac operator to be defined in Sec. II-in all of these phenomena is suggestive. We hope that the resulting infrared-ultraviolet connection we find in this work will fill missing logical gaps in related discussions.

For the most part in this paper, we will employ the heat kernel method [50], as it is universally applicable to all spacetime dimensions and is very effective for extracting topological information. In Sec. IV, however, we will also resort to the usual triangular Feynman diagram for the $d=4$ chiral anomaly, where the modification of the current operators, on top of the higher inverse power of the propagator, plays a crucial role.

\section{GENERALIZED SPINORS AND DIRAC OPERATORS}

We would like to consider a Dirac index problem with the operator generalized as

\footnotetext{
${ }^{1}$ See Refs. [41-43] for the experimental observation of the chiral magnetic effect in Weyl semimetals, and see Refs. [44,45] for the recent status of the experimental search of the chiral magnetic effect in relativistic heavy-ion experiments.
} 


$$
\mathcal{Q}=\gamma^{\mu} \mathcal{P}_{\mu}(-i D)
$$

with smooth functions $\mathcal{P}_{\mu}$. Let us take the Dirac matrices in the chiral basis,

$$
\gamma^{a}=\left(\begin{array}{cc}
0 & \sigma^{a} \\
\sigma^{a} & 0
\end{array}\right), \quad \gamma^{4}=\left(\begin{array}{cc}
0 & -i \\
i & 0
\end{array}\right),
$$

and we use the covariant derivative

$$
D_{\mu}=\partial_{\mu}+A_{\mu}
$$

with the anti-Hermitian gauge field $A_{\mu}$. The Dirac operator has the form

$$
\mathcal{Q}=\left(\begin{array}{cc}
0 & \mathcal{D} \\
\mathcal{D}^{\dagger} & 0
\end{array}\right)
$$

with

$$
\mathcal{D}=\left(\sigma^{a} \mathcal{P}_{a}-i \mathcal{P}_{4}\right), \quad \mathcal{D}^{\dagger}=\left(\sigma^{a} \mathcal{P}_{a}+i \mathcal{P}_{4}\right) .
$$

We are interested in the index theorem of $\mathcal{Q}$, and the chiral anomaly associated with a Weyl fermion with the kinetic operator $\mathcal{D}$.

\section{A. Index density and chiral anomaly}

As is well known from the Fujikawa method [3], the failure of the chiral rotation of the path integral for the relevant two-component Weyl fermion,

$$
\int[D \bar{\psi} D \psi] e^{\int \bar{\psi} \mathcal{D} \psi},
$$

is measured by the index density, which can be written formally as

$$
\operatorname{Tr}(\Gamma) \equiv \lim _{s \rightarrow 0} \operatorname{Tr}\left(\Gamma e^{-s \mathcal{Q}^{2}}\right),
$$

with $\Gamma=-\gamma^{1} \gamma^{2} \gamma^{3} \gamma^{4}$. Note that the trace here is over the four-component Dirac spinors even though the physical system is that of a Weyl spinor. One can understand this from the well-known fact that, in the Euclidean signature, $\bar{\psi}$ has to be treated as independent and transforms oppositely to $\psi$ under the chiral rotation. In practice, $\psi$ and $\bar{\psi}$ together define a Dirac spinor, for which the formal index problem follows. For a most comprehensive study of anomaly and the connection to the index theorem, we refer readers to Ref. [51].

Here, we proceed to compute this quantity by modifying the usual heat kernel method, or

$$
\lim _{s \rightarrow 0} \operatorname{Tr}\left(\Gamma e^{-s \mathcal{Q}^{2}}\right)=\lim _{s \rightarrow 0} \int d^{4} x \operatorname{tr}\left(\Gamma G_{s}(x ; x)\right),
$$

where $G_{s}(y ; x) \equiv\left\langle y\left|e^{-s \mathcal{Q}^{2}}\right| x\right\rangle$ obeys

$$
-\partial_{s} G_{s}(y ; x)=\mathcal{Q}^{2} G_{s}(y ; x), \quad \lim _{s \rightarrow 0} G_{s}(y ; x)=\delta^{(4)}(y-x) .
$$

So, the problem boils down to how one computes $G_{s}(x ; x)$. For this, we start with

$$
\mathcal{Q}^{2}=\mathcal{P}_{\mu} \mathcal{P}_{\mu}+\frac{1}{4}\left[\gamma^{\mu} \gamma^{\nu}\right]\left[\mathcal{P}_{\mu}, \mathcal{P}_{\nu}\right]
$$

which we further split as

$$
\mathcal{Q}^{2}=\mathcal{Q}_{0}^{2}+\delta \mathcal{Q}^{2}, \quad Q_{0}^{2} \equiv \mathcal{P}_{\mu}(-i \partial) \mathcal{P}_{\mu}(-i \partial) .
$$

With the latter, we can perform the usual heat kernel expansion

$$
\begin{aligned}
G_{s}(y ; x) & =\sum_{l=0} G_{s}^{(l)}(y ; x), \\
G_{s}^{(l+1)}(y ; x) & =-\int_{0}^{s} d t \int d^{4} z G_{s-t}^{(0)}(y ; z) \delta \mathcal{Q}^{2} G_{t}^{(l)}(z ; x),
\end{aligned}
$$

where the free heat kernel

$$
G_{s}^{(0)}(z ; x)=\left\langle z\left|e^{-s \mathcal{Q}_{0}^{2}}\right| x\right\rangle,
$$

which is easily found,

$$
G_{s}^{(0)}(x+X ; x)=\int \frac{d^{4} K}{(2 \pi)^{4}} e^{i K \cdot X} e^{-s \mathcal{P}(K)^{2}},
$$

in the momentum space $\tilde{\mathbb{R}}^{4}$ of $K_{\mu}$.

For the index density, the crucial step is the powercounting of small $s$ in Eq. (2.12). Each iteration brings down a factor of $s$ given the $s$ integral, but further fractional factors of $s$ arise from the $z$ integral combined with operators in $\delta \mathcal{Q}^{2}$. Note, in particular, that each derivative in $\delta \mathcal{Q}^{2}$ will cost some inverse fractional power of $s$. One key identity will be

$$
\frac{1}{\sqrt{\pi^{4}}} \int d^{4} K \operatorname{det}\left(\frac{\partial \mathcal{P}_{\mu}}{\partial K_{\alpha}}\right) e^{-s \mathcal{P}(K)^{2}}=s^{-2} N_{\mathcal{P}}
$$

where $N_{\mathcal{P}}$ is the asymptotic winding number of the map, $K \rightarrow \mathcal{P}(K)$. In other words, $N_{\mathcal{P}}$ measures the multiplicity of the map over the target $\mathbb{R}^{4}$ with the orientation taken into account. With Eqs. (2.10) and (2.11), and with the insertion of $\Gamma$ in Eq. (2.7), it is clear that the first nontrivial expression out of Eq. (2.12) will occur at the second iteration, where we expect to find something like Eq. (2.15) 
times $s^{2}$, leading us to $N_{\mathcal{P}}$ in the end. Let us now track how this occurs.

The relevant contribution can be found from the further expansion of the squared Dirac operator

$$
\frac{1}{4}\left[\gamma^{\mu}, \gamma^{\nu}\right]\left[\mathcal{P}_{\mu}, \mathcal{P}_{\nu}\right]=\frac{1}{2} \gamma^{\mu} \gamma^{\nu} F_{\alpha \beta} \frac{\partial \mathcal{P}_{\mu}(K)}{\partial K_{\alpha}} \frac{\partial \mathcal{P}_{\nu}(K)}{\partial K_{\beta}}+\cdots,
$$

where the ellipsis denotes terms that come with fewer freestanding derivatives-i.e., fewer factors of $K$, or more $A$ 's. These cost lower powers of $s^{-1}$ and effectively disappear as the $s \rightarrow 0$ limit is taken in the end. Because of the $\Gamma$ insertion in Eq. (2.7), the first nontrivial term arises in the second order of the iteration of Eq. (2.12), when one pulls down $\gamma \gamma F$ in Eq. (2.16). This will be accompanied effectively by a factor of $s^{2} / 2$, due to the two $s$ integrals, producing a term like Eq. (2.15). This shows how all the subsequent terms in Eq. (2.16) become irrelevant for the purpose of computing the index density. In fact, all interaction pieces in $\mathcal{P}(-i D)^{2}$ belong to the latter category, so for the purpose of computing the index density, all that matters is the first term on the right-hand side of Eq. (2.16) in the place of $\delta \mathcal{Q}^{2}$.

Let us trace this process more explicitly. Since an explicit factor of $x$ 's in $\delta \mathcal{Q}^{2}$, such as in the Taylor expansion of $F$, costs positive factors of $s$, relative to the one in Eq. (2.16), we only need to worry about how the free-standing derivatives in $\delta \mathcal{Q}^{2}$ works in the heat kernel expansion. With

$$
\begin{aligned}
\Pi(K ; F) & \equiv \frac{1}{2} \gamma^{\mu} \gamma^{\nu} F_{\alpha \beta} \frac{\partial \mathcal{P}_{\mu}(K)}{\partial K_{\alpha}} \frac{\partial \mathcal{P}_{\nu}(K)}{\partial K_{\beta}}, \\
\hat{\Pi}(-i \partial ; F) & \left.\equiv \Pi(K ; F)\right|_{K \rightarrow-i \partial},
\end{aligned}
$$

one finds ${ }^{2}$

$$
\begin{aligned}
G_{s}^{(1)}(x+X ; x) & =\int_{0}^{s} d t \int d^{4} Y G_{s-t}^{(0)}(x+X ; x+Y) \hat{\Pi}(-i \partial ; F) G_{t}^{(0)}(x+Y ; x)+\cdots \\
& =\int_{0}^{s} d t \int d^{4} Y \int \frac{d^{4} K}{(2 \pi)^{4}} e^{i K \cdot(X-Y)} e^{-(s-t) \mathcal{P}(K)^{2}} \times \Pi(W ; F) \int \frac{d^{4} W}{(2 \pi)^{4}} e^{i W \cdot Y} e^{-t \mathcal{P}(W)^{2}}+\cdots \\
& =s \int \frac{d^{4} K}{(2 \pi)^{4}} e^{i K \cdot X} e^{-s \mathcal{P}(K)^{2}} \times \Pi(K ; F(x))+\cdots
\end{aligned}
$$

The above is from an expansion of the operator $\mathcal{Q}^{2}$ around a generic point $x$, and the momentum $K$ is conjugate to the "small" displacement $X$.

It is clear that the momentum factors pile up through the iteration, and since the position dependence of $F_{\mu \nu}(x)$ does not enter in the small-s limit, the iteration can be performed in the momentum space straightforwardly. Repeating one more time, the same computation gives

$$
\begin{aligned}
G_{s}^{(2)}(x+X ; x)= & \frac{s^{2}}{2} \int \frac{d^{4} K}{(2 \pi)^{4}} e^{i K \cdot X} e^{-s \mathcal{P}(K)^{2}} \\
& \times(\Pi(K ; F(x)))^{2}+\cdots
\end{aligned}
$$

Now we are ready to compute the index density:

\footnotetext{
${ }^{2}$ Here we use

$$
\int d^{4} Y e^{i(W-K) \cdot Y}=(2 \pi)^{4} \delta^{(4)}(W-K) .
$$
}

$$
\begin{aligned}
\lim _{s \rightarrow 0} \operatorname{Tr}\left(\Gamma e^{-s \mathcal{Q}^{2}}\right)= & \lim _{s \rightarrow 0} \int d^{4} x \operatorname{tr}\left(\Gamma G_{s}(x ; x)\right) \\
= & \lim _{s \rightarrow 0} \frac{s^{2}}{2} \int d^{4} x \operatorname{tr}\left(\frac{1}{4} \Gamma \gamma^{\mu} \gamma^{\nu} \gamma^{\mu^{\prime}} \gamma^{\nu^{\prime}}\right) F_{\alpha \beta} F_{\alpha^{\prime} \beta^{\prime}} \\
& \times \int \frac{d^{4} K}{(2 \pi)^{4}} \frac{\partial \mathcal{P}_{\mu}(K)}{\partial K_{\alpha}} \frac{\partial \mathcal{P}_{\nu}(K)}{\partial K_{\beta}} \\
& \times \frac{\partial \mathcal{P}_{\mu^{\prime}}(K)}{\partial K_{\alpha^{\prime}}} \frac{\partial \mathcal{P}_{\nu^{\prime}}(K)}{\partial K_{\beta^{\prime}}} e^{-s \mathcal{P}(K)^{2}}
\end{aligned}
$$

With $\Gamma=-\gamma^{1} \gamma^{2} \gamma^{3} \gamma^{4}$ inserted, we need to collect four distinct $\gamma$ 's to ensure a nonzero result, and then the fermionic trace above gives $-4 \epsilon^{\mu \nu \mu^{\prime} \nu^{\prime}}$. Then, we may invoke Eq. (2.15) and find

$$
\begin{aligned}
\lim _{s \rightarrow 0} \operatorname{Tr}\left(\Gamma e^{-s \mathcal{Q}^{2}}\right) & =-\frac{N_{\mathcal{P}}}{32 \pi^{2}} \int d^{4} x \epsilon^{\alpha \beta \alpha^{\prime} \beta^{\prime}} F_{\alpha \beta} F_{\alpha^{\prime} \beta^{\prime}} \\
& =N_{\mathcal{P}} \cdot\left(-\frac{1}{8 \pi^{2}} \int F \wedge F\right),
\end{aligned}
$$

which shows the usual index density, and hence the chiral anomaly is enhanced by a factor of $N_{\mathcal{P}}$, the winding number associated with the map $K \rightarrow \mathcal{P}(K)$. 
Although we have computed the index density in four dimensions, the generalization to arbitrary even dimensions, $d$, is immediate, and gives

$$
\lim _{s \rightarrow 0} \operatorname{Tr}\left(\Gamma e^{-s \mathcal{Q}^{2}}\right)=N_{\mathcal{P}} \cdot\left(\frac{1}{(d / 2) !(2 \pi i)^{d / 2}} \int F \wedge \cdots \wedge F\right),
$$

with a $d / 2$ number of the field strength 2-form $F=F_{\mu \nu} d x^{\mu} \wedge d x^{\nu} / 2$, anti-Hermitian as before. The winding number of the map $K \rightarrow \mathcal{P}(K)$ enters this formula via ${ }^{3}$

$$
N_{\mathcal{P}} \equiv \frac{1}{\sqrt{\pi}} \int_{\mathbb{R}^{d}} d^{d} K \operatorname{det}\left(\tilde{\partial}^{\alpha} \mathcal{P}_{\mu}\right) e^{-\mathcal{P}(K)^{2}}
$$

Each oriented copy of $\hat{\mathbb{R}}^{d}$ in the image gives 1 times the sign of the Jacobian, so this measures how many times the map $\mathcal{P}(K)$ covers the target $\hat{\mathbb{R}}^{d}$. If the map is such that $\mathcal{P}(K)^{2}$ diverges wherever $K^{2}$ diverges, this number $N_{\mathcal{P}}$ is an integer.

A simple class of illuminating examples can be found for $d=2$. The winding number $N_{\mathcal{P}}$ would be the net multiplicity of the map $\mathcal{P}: \tilde{\mathbb{R}}^{2} \rightarrow \hat{\mathbb{R}}^{2}$, or equivalently, the net winding number of the asymptotic circle in $\tilde{\mathbb{R}}^{2}$. For instance, consider the special case of $\mathcal{P}=\tilde{\partial} W$ and $W=$ $\left(f+f^{*}\right)$ for some holomorphic polynomial $f=f\left(K_{1}+\right.$ $i K_{2}$ ) of the maximal degree $n+1$. The winding number can be extracted easily by considering

$$
\mathcal{P}_{1}+i \mathcal{P}_{2}=\left(\tilde{\partial}_{1}+i \tilde{\partial}_{2}\right)\left(f+f^{*}\right)=2 \partial_{K_{1}-i K_{2}} f^{*}
$$

along the asymptotic circle; it is proportional to $\left(K_{1}-\right.$ $\left.i K_{2}\right)^{n}$ asymptotically, so we learn $N_{\mathcal{P}}=-n$ regardless of the details of $f$. If we generalize $\left(f+f^{*}\right)$ to an arbitrary real polynomial $W\left(K_{1,2}\right)$, again of the highest degree $n+1$, as the next logical step, we already lose such a universal statement. The same mathematical object $N_{\mathcal{P}=\tilde{\partial} W}$ has been recently studied in an entirely different context [52]. There, the authors found $N_{\mathcal{P}=\tilde{\partial} W}=-n,-n+2,-n+4, \ldots$, etc., provided that the leading power of $W$ is nondegenerate, with codimension- 1 walls dividing the parameter space of $W$ into domains with locally constant $N_{\mathcal{P}=\tilde{\partial} W}$. In other words, despite the "topological" nature, $N_{\mathcal{P}}$ can experience "wall crossing" generically. We refer the readers to Sec. 2 of Ref. [52] for a full range of subtleties, present even for this simple class of examples, such as (non)integrality criteria and wall crossings thereof.

\footnotetext{
${ }^{3}$ We introduce $\tilde{\partial}$ to emphasize that it is a partial derivative in the momentum space.
}

\section{B. Infrared interpretation}

Note that this integral produces an integer, as long as $\mathcal{P}^{2}$ is asymptotically unbounded, since

$$
N_{\mathcal{P}}=\frac{1}{\sqrt{\pi}^{d}} \int d^{d} \mathcal{P} e^{-\mathcal{P}^{2}},
$$

where $N_{\mathcal{P}}$ is now entirely encoded in the integration domain. The integral on the right-hand side gives 1 for each integration domain of $\hat{\mathbb{R}}^{d}$, but this is multiplied by $N_{\mathcal{P}}$, since the map $K \rightarrow \mathcal{P}$ is an $N_{\mathcal{P}}$-fold covering of $\hat{\mathbb{R}}^{d}$. This topological characterization may be considered an ultraviolet description, since the winding number is defined via the asymptotic behavior of the map $\mathcal{P}(K)$.

On the other hand, the index and the anomaly are fundamentally infrared phenomena, so they should be equally visible in the small- $|\mathcal{P}|$ limit. For this, note that the expression is invariant under $\mathcal{P} \rightarrow C \cdot \mathcal{P}$ for any positive real number $C$, which we already used to scale away $s$ above to reach Eq. (2.24). Going back to the $K$-space integral and taking a limit of $C \rightarrow \infty$, however, we see that Eq. (2.24) localizes at the critical points, $\mathcal{P}=0$, and the winding number has an alternative form, as a sum over the critical points, weighted by \pm 1 , depending on the sign of the determinant there:

$$
\left.\sum_{\left\{K_{*} \mid \mathcal{P}\left(K_{*}\right)=0\right\}} 1 \cdot \operatorname{sgn}\left[\operatorname{det}\left(\tilde{\partial}^{\alpha} \mathcal{P}_{\mu}\right)\right]\right|_{K=K_{*}},
$$

provided that all the critical points are nondegenerate-i.e., provided that the determinants there do not vanish. In fact, it is easy to see how this generalizes to a case with degenerate critical points,

$$
\sum_{\left\{K_{*} \mid \mathcal{P}\left(K_{*}\right)=0\right\}} N_{\mathcal{P}}\left(K_{*}\right),
$$

where $N_{\mathcal{P}}\left(K_{*}\right)$ is the local winding number near such (degenerate) critical points. This is a Morse theory counting if $\mathcal{P}_{\mu}=\tilde{\partial}_{\mu} W(K)$ for some Morse function $W(K)$ [9], although we do not really need the latter here.

Again, let us consider the minimal example we saw in the previous subsection, with $W=f+f^{*}$ for a holomorphic polynomial $f\left(K_{1}+i K_{2}\right)$ of the highest degree $n+1$ in $d=2$. The critical points $K_{*}$ are found by solving $f^{\prime}=0$, which is a degree- $n$ algebraic equation on $\mathbb{C}$, with $n$ solutions. Near a simple zero-say, $K_{*}-f$ can always be approximated by a quadratic term $f-f\left(K_{*}\right) \simeq$ $C\left(\Delta K_{1}+i \Delta K_{2}\right)^{2}$, so that $W-W\left(K_{*}\right) \simeq|2 C|\left(\left(\Delta K_{1}\right)^{2}-\right.$ $\left.\left(\Delta K_{2}\right)^{2}\right)$ modulo a local rotation of $\Delta K \equiv K-K_{*}$. The sign of the Hessian is always negative at $K_{*}$, so we again find $N_{\mathcal{P}}=-n$, the same as above from the winding number interpretation. With $W$ elevated to an arbitrary real polynomial, the number of zeros becomes more flexible, with 
both signs of the Hessian allowed. In the end, one finds agreement [52] between this fixed-point counting and the winding number counting of the previous subsection.

Although we started with the expression (2.24) that has a natural interpretation as a winding number, measured at the asymptotic region of $K$ space, this alternative description counts the critical points, $\mathcal{P}=0$, where the Dirac operator $\mathcal{Q}$ may be approximated by a linear form,

$$
\mathcal{Q} \simeq \tilde{\partial}^{\alpha} \mathcal{P}_{\mu}\left(K_{*}\right)\left(-i \gamma^{\mu} D_{\alpha}\right) .
$$

One merely counts how many approximate Dirac cones appear in the infrared end of the dynamics, whose chiralities are dictated by the matrix $\tilde{\partial}^{\alpha} \mathcal{P}_{\mu}\left(K_{*}\right)$. If the latter has a negative determinant, this can be translated to a chirality flip, relative to others with a positive determinant.

\section{NONRELATIVISTIC ISOSPINOR}

For condensed matter systems, one sometimes encounters generalized Weyl fermions where the two components actually refer to flavors or "isospin" rather than the real spin associated with the angular momentum. In recent years, the chiral anomaly in this isospin context has surfaced as an important issue, so let us apply what we have developed in the previous section to these real systems.

Since these are all nonrelativistic fermions, the direction 4 plays a special role as the genuine (Euclidean) time direction, and as such, we will be content with the single derivative there. As such, we may specialize to the case

$$
\mathcal{P}_{4}=-i D_{4}+\Delta(-i \vec{D}), \quad \mathcal{P}_{a}=P_{a}(-i \vec{D}),
$$

where we split the 4-vector into the Euclidean time component and a 3-vector distinguished by the arrow. This will correspond, in Lorentzian time, to a two-component Weyl Hamiltonian of type

$$
H=\sigma^{a} P_{a}-\Delta .
$$

The necessary Wick rotation prescription will become clearer when we compute the anomaly by a Feynman diagram in the next section, but for now we will stick to this Euclidean viewpoint.

We could simply rely on the results of the previous section, whereby the anomaly can be seen to not be affected by the presence of $\Delta$ at all. Still, let us retrace part of these steps for an illustration, with a simplifying assumption of $\Delta=0$. In other words, let us consider

$\mathcal{D}=\sigma^{a} P_{a}(-i \vec{D})-D_{4}, \quad \mathcal{D}^{\dagger}=\sigma^{a} P_{a}(-i \vec{D})+D_{4}$,

for some smooth functions $P_{a}$ of $-i D_{a=1,2,3}$. In addition, we will assume that $|\vec{P}(\vec{k})|^{2}$ grows indefinitely at large $\vec{k}$; this would be the case, for example, if $P$ 's are generic polynomials. Our experience above suggests that the anomaly must be again dictated by a topology of the $\operatorname{map} k_{a} \rightarrow P_{a}(\vec{k})$.

The zeroth-order heat kernel is

$G_{s}^{(0)}(x+X ; x)=\frac{1}{\sqrt{4 \pi s}} e^{-X_{4}^{2} / 4 s} \times \int \frac{d^{3} k}{(2 \pi)^{3}} e^{i \vec{k} \cdot \vec{X}} e^{-s \vec{P}^{2}}$,

where we now separate out the 3-vector $\vec{X}$ from the 4-vector $X$, etc. The pieces in $\mathcal{Q}^{2}$ that can contribute to the index density are, as before,

$$
\begin{aligned}
\frac{1}{4}\left[\gamma^{\mu}, \gamma^{\nu}\right]\left[\mathcal{P}_{\mu}, \mathcal{P}_{\nu}\right]= & \sum_{a=1}^{3} \gamma^{4} \gamma^{a} F_{4 h} \tilde{\partial}^{h} P_{a}(\vec{k})+\cdots \\
& +\sum_{b>c} \gamma^{b} \gamma^{c} F_{f g} \tilde{\partial}^{f} P_{b}(\vec{k}) \tilde{\partial}^{g} P_{c}(\vec{k})+\cdots,
\end{aligned}
$$

expressed in the momentum space, and $F$ is considered to be slowly varying. The structure of the index density we have seen implies that the integrand will contain the multiplicative factor

$\frac{1}{6} \epsilon^{a b c} \epsilon_{h f g}\left(\tilde{\partial}^{h} P_{a}\right)\left(\tilde{\partial}^{f} P_{b}\right)\left(\tilde{\partial}^{g} P_{c}\right)=\operatorname{det}\left(\frac{\partial P_{a}(\vec{k})}{\partial k_{h}}\right)$,

and we can deduce the expression that plays the role of $N_{\mathcal{P}}$ in Eq. (2.22):

$N_{\vec{P}} \equiv \frac{s^{3 / 2}}{\sqrt{\pi}^{3}} \int d^{3} k \operatorname{det}\left(\frac{\partial P_{a}(\vec{k})}{\partial k_{h}}\right) e^{-s \vec{P}^{2}}=\frac{1}{\sqrt{\pi^{3}}} \int d^{3} P e^{-\vec{P}^{2}}$,

where the factor $s$ on the left-hand side is scaled away without affecting the result by the change of the integration variable $s^{1 / 2} \vec{P} \rightarrow \vec{P}$ on the right-hand side.

Again, the map $\vec{k} \rightarrow \vec{P}(\vec{k})$ is in general a multiple cover of the target $\hat{\mathbb{R}}^{3}$, which translates to the domain of the integral on the right-hand side being several copies of $\hat{\mathbb{R}}^{3}$ : The integral measures precisely this multiplicity. Alternatively, this can be viewed as the winding number of the map $\vec{k} /|\vec{k}| \rightarrow \vec{P}(\vec{k}) /|P(\vec{k})|$, from $\tilde{\mathbb{S}}^{2}$ to $\hat{\mathbb{S}}^{2}$, in the large- $|\vec{k}|$ limit. We conclude that, for the most general nonrelativistic Weyl fermion in $3+1$ dimensions, Eq. (3.7) is the right coefficient to the chiral anomaly.

\section{A. The Berry monopoles in the momentum space}

We saw that the chiral anomaly of a general twocomponent isospinor is given by the winding number of the map $\vec{k} \rightarrow \vec{P}(\vec{k})$ in momentum space. We will show that 
the same topology underlies the Berry connection of the projected chiral spinor in momentum space. ${ }^{4}$ For this, one considers a two-level problem with the Hamiltonian

$$
\sigma^{a} P_{a}(\vec{k})
$$

whose two eigenvalues are $\pm|\vec{P}(\vec{k})|$. Denoting the two respective eigenvectors by $| \pm\rangle$, the Berry connection is

$$
\mathcal{A}^{ \pm}=-\left\langle \pm\left|\frac{\partial}{\partial k_{a}}\right| \pm\right\rangle d k_{a} .
$$

A well-known result is that, when $P_{a}=k_{a}$, the Berry connection carries the unit magnetic flux. Let us recapitulate this simpler case first. In the spherical coordinates,

$$
\sigma^{a} k_{a}=|\vec{k}|\left(\begin{array}{cc}
\cos \theta & \sin \theta e^{-i \phi} \\
\sin \theta e^{i \phi} & -\cos \theta
\end{array}\right),
$$

the two eigenvectors are

$$
\begin{aligned}
|+\rangle_{\sigma^{a} k_{a}} & =\left(\begin{array}{c}
\cos (\theta / 2) e^{-i \phi / 2} \\
\sin (\theta / 2) e^{i \phi / 2}
\end{array}\right), \\
|-\rangle_{\sigma^{a} k_{a}} & =\left(\begin{array}{c}
-\sin (\theta / 2) e^{-i \phi / 2} \\
\cos (\theta / 2) e^{i \phi / 2}
\end{array}\right) .
\end{aligned}
$$

Therefore, the two Berry connections are common up to a sign,

$$
\left.\mathcal{A}^{ \pm}\right|_{\sigma^{a} k_{a}}= \pm \frac{i}{2} \cos \theta d \phi
$$

which carries a unit $2 \pi$ magnetic flux over $\tilde{\mathbb{S}}^{2}$, or the unit Chern number. As usual, this is up to $U(1)$ gauge transformations under the innocuous phase rotations,

$$
| \pm\rangle \rightarrow e^{i \Lambda_{ \pm}(\vec{k})}| \pm\rangle
$$

Coming back to $H=\sigma^{a} P_{a}$ is merely a matter of replacing $\vec{k}$ with $\vec{P}(\vec{k})$, so we can express

$$
\left.\mathcal{A}^{ \pm}\right|_{\sigma^{a} P_{a}}= \pm \frac{i}{2} \cos \Theta d \Phi
$$

with the spherical angles $\Theta$ and $\Phi$ of $\vec{P}$, which needs to be pulled back to $\vec{k}$ space. In other words, denoting the standard $2 \pi$ Wu-Yang monopole field [53] in $\vec{P}$ space by $\mathbf{A}$

\footnotetext{
${ }^{4}$ What we outline here is a well-known computation in the context of $d=2+1$ topological insulators generally. See, for example, Ref. [16]. One way to realize the latter is by imagining a two-dimensional Brillouin zone as a slice in a $d=3+1$ Brillouin zone of Weyl semimetal, between a pair of chiral Weyl and antichiral Weyl points.
}

and its field strength by $\mathbf{F}$, the Berry connection and the field strength thereof in the momentum space are

$$
\begin{aligned}
\mathcal{A}^{ \pm} & = \pm \mathbf{A}_{a} \frac{\partial P^{a}}{\partial k_{f}} d k_{f}, \\
\mathcal{F}^{ \pm} & = \pm \frac{1}{2} \mathbf{F}_{a b} \frac{\partial P^{a}}{\partial k_{f}} \frac{\partial P^{b}}{\partial k_{g}} d k_{f} \wedge d k_{g} .
\end{aligned}
$$

Although Eq. (3.14) seemingly gives a unit $2 \pi$ flux, this is not generally the case, because the map $\vec{k} \rightarrow \vec{P}$ can be a multicover of the target $\hat{\mathbb{R}}^{3}$.

The actual integral that computes the Chern number is, with $P^{*}$ being the pullback of the map $\vec{P}(\vec{k})$,

$$
\frac{1}{2 \pi i} \int_{S_{2}} \mathcal{F}^{ \pm}= \pm \frac{1}{2 \pi} \int_{S_{2}} P^{*}(\mathbf{F})= \pm \frac{1}{2 \pi} \int_{B_{3}} P^{*}(d \mathbf{F}),
$$

where $S_{2}=\partial B_{3}$ is an arbitrary 2-surface in the $\vec{k}$ space. In particular, if one takes $B_{3}=\tilde{\mathbb{R}}^{3}$, the entire momentum space, then

$$
\frac{1}{2 \pi i} \int_{\tilde{\mathbb{R}}^{3}} P^{*}(d \mathbf{F})=\frac{1}{2 \pi i} \oint_{\tilde{\mathbb{S}}_{\infty}^{2}} P^{*}(\mathbf{F})=N_{\vec{P}}
$$

since at the asymptotic two-sphere $\tilde{\mathbb{S}}_{\infty}^{2}$, the total flux is multiplied by the net winding number $N_{\vec{P}}$.

On the other hand, $d \mathbf{F}=0$ everywhere except at the origin $\vec{P}=0$, so we may rewrite this integral as

$$
\frac{1}{2 \pi} \sum_{\left\{\vec{k}_{*} \mid \vec{P}\left(\vec{k}_{*}\right)=0\right\}} \int_{B_{\varepsilon}^{3}\left(\vec{k}_{*}\right)} P^{*}(d \mathbf{F}),
$$

where $B_{\epsilon}^{3}\left(\vec{k}_{*}\right)$ is an infinitesimal 3-ball centered at $\vec{k}_{*}$. This in turn becomes

$$
\frac{1}{2 \pi i} \sum_{\left\{\vec{k}_{*} \mid \vec{P}\left(\vec{k}_{*}\right)=0\right\}} \int_{\partial B_{\epsilon}^{3}\left(\vec{k}_{*}\right)} P^{*}(\mathbf{F}),
$$

and, since $\mathbf{F}$ is a unit monopole in $\vec{P}$ space, we find

$$
\sum_{\left\{\vec{k}_{*} \mid \vec{P}\left(\vec{k}_{*}\right)=0\right\}} N_{\vec{P}}\left(\vec{k}_{*}\right)=N_{\vec{P}},
$$

where $N_{\vec{P}}\left(\vec{k}_{*}\right)$ is the winding number of $\vec{P}$, measured by the infinitesimal neighborhood around $\vec{k}_{*}$, which of course sums up to $N_{\vec{P}}$.

The total flux is computed by taking $S_{2}$ equal to the asymptotic $\tilde{\mathbb{S}}^{2}$ of $\vec{k}$ space, $\tilde{\mathbb{R}}^{3}$, which maps to $N_{\vec{P}}$ times the asymptotic $\hat{\mathbb{S}}^{2}$ of the target $\hat{\mathbb{R}}^{3}$ if the map $\vec{P}$ covers the target $\hat{\mathbb{R}}^{3} N_{\vec{P}}$ times. As such, this Berry connection carries precisely $2 \pi N_{\vec{P}}$ total flux, where $N_{\vec{P}}$ may be also computed 
as in Eq. (3.7), provided that $\vec{P}^{2}$ is divergent everywhere asymptotically. This shows that the chiral anomaly is precisely given by the same winding number $N_{\vec{P}}$, offering a rigorous logical link between the chiral anomaly and the Berry connection in momentum space. As should be clear from how we arrive at this connection via our generalized index theorem, we emphasize that this is a nontrivial, yet understandable manifestation of the infrared-ultraviolet connection, which is a fundamental characteristic of the chiral anomaly.

\section{B. Infrared view and real material}

As we have already seen in Sec. II, one can compute the same winding number alternatively by rescaling $P_{a} \rightarrow$ $C \cdot P_{a}$, and going back to a $d^{3} k$ integral. This localizes to small neighborhoods around $\vec{k}=\vec{k}_{*}$ where $\vec{P}\left(\vec{k}_{*}\right)=0$, and as such really counts inverse images of $\vec{P}=0$ with weights \pm 1 ,

$$
\left.\sum_{\left\{\vec{k}_{*} \mid \vec{P}\left(\vec{k}_{*}\right)=0\right\}} 1 \cdot \operatorname{sgn}\left[\operatorname{det}\left(\tilde{\partial}^{h} P_{a}\right)\right]\right|_{\vec{k}=\vec{k}_{*}} \cdot
$$

This alternative form is viable when the critical points are isolated and nondegenerate, where one is counting Weyl cones with $\vec{P}$ locally linear $\vec{k}$. If the sign happens to be negative, it has the same effect as flipping the signs of an odd number of $\gamma^{a}$ 's, resulting in anti-Weyl fermions instead of Weyl fermions. When we allow degenerate critical points, we find

$$
\sum_{\left\{\vec{k}_{*} \mid \vec{P}\left(\vec{k}_{*}\right)=0\right\}} N_{\vec{P}}\left(\vec{k}_{*}\right),
$$

the same as Eq. (3.20).

In real material, the momentum $\vec{k}$ lives in a compact Brillouin zone, $\tilde{\mathbb{R}}^{3} / \Lambda$, where $\Lambda$ is the dual lattice to the crystalline lattice of the material. On the flip side, $\vec{P}$ does not extend indefinitely into $\hat{\mathbb{R}}^{3}$ either. Instead, $\vec{P}(\vec{k})=$ $\vec{P}(\Lambda \vec{k})$. One immediate question is how the story so far is affected by such a compact Brillouin zone. Consider a pair of energy bands that meets at one or more Bloch momentum $\vec{k}=\vec{k}_{*}$, which for our purpose means a continuous map from $\vec{k}$ in the Brillouin zone $\tilde{\mathbb{R}}^{3} / \Lambda$ to a $2 \times 2$ Hamiltonian $H(\vec{k})$, such that

$$
H(\vec{k})=\vec{\sigma} \cdot \vec{P}(\vec{k})-\Delta(\vec{k}), \quad \vec{P}\left(\vec{k}_{*}\right)=0 .
$$

The appearance of $\Delta$ is of course necessary for real material, but its connection to the same symbol in the above Euclidean computation might look a bit unclear, since the naive Wick rotation would render $\Delta$ in Eq. (3.1) to become purely imaginary. The answer to this is that one really starts with a Lorentzian signature and rotates the contour of $k_{0}$ such that one reaches Eq. (3.1) in the end. More on this will be elaborated in the next section.

In both the Euclidean anomaly computation and the Berry phase computation, we have seen that only the winding number associated with $\vec{P}(\vec{k})$ matters for these topological characterizations. In the target, one starts with a monopole of the Berry connection near $\vec{P}=0$, which we must pull back to the Brillouin zone $\tilde{\mathbb{R}}^{3} / \Lambda$. One puzzling aspect is that now $\vec{P}^{2}$ must be bounded, as it is defined on a compact Brillouin zone to begin with, and in real material the energy eigenvalues $-\Delta \pm|\vec{P}|$ must be bounded above and below: the integral formulas such as Eq. (3.7) appear to have no reason to produce an integer. This quandary is saved by the observation that under such circumstances, (3.7) always produces zero, as we see below.

Let us replace $\tilde{\mathbb{R}}^{3}$ with $\tilde{\mathbb{R}}^{3} / \Lambda$ and consider a surface $S_{2}=\partial B_{3}$ that encloses all the critical points $P\left(\vec{k}_{*}\right)=0$, whereby we have

$$
N_{\vec{P}}=\frac{1}{2 \pi i} \int_{B_{3}} d P^{*}(\mathbf{F})=\frac{1}{2 \pi i} \int_{S_{2}} P^{*}(\mathbf{F}) .
$$

On the other hand, since $\tilde{\mathbb{R}}^{3} / \Lambda$ is closed, $-S_{2}$ is also a boundary to the complement $B_{3}^{c}$. Given that no magnetic monopole exists in $B_{3}^{c}$, we have

$N_{\vec{P}}=-\frac{1}{2 \pi i} \int_{-S_{2}} P^{*}(\mathbf{F})=-\frac{1}{2 \pi i} \int_{B_{3}^{c}} d P^{*}(\mathbf{F})=0$,

and further may as well shrink $B_{3}^{c}$ to nothing, so that $B_{3}=\tilde{\mathbb{R}}^{3} / \Lambda$, and find

$$
N_{\vec{P}}=\frac{1}{2 \pi} \int_{\tilde{\mathbb{R}}^{3} / \Lambda} d\left(P^{*}(\mathbf{F})\right)=0 .
$$

In view of our infrared alternative, the same can be expressed as

$$
\sum_{\left\{\vec{k}_{*} \mid \vec{P}\left(\vec{k}_{*}\right)=0\right\}} N_{\vec{P}}\left(\vec{k}_{*}\right)=0
$$

or

$$
\left.\sum_{\left\{\vec{k}_{*} \mid \vec{P}\left(\vec{k}_{*}\right)=0\right\}} 1 \cdot \operatorname{sgn}\left[\operatorname{det}\left(\tilde{\partial}^{h} P_{a}\right)\right]\right|_{\vec{k}=\vec{k}_{*}}=0,
$$

if all the critical points are nondegenerate.

In the end, the number of Weyl points and the number of anti-Weyl points are always equal, for any system on a real space lattice, provided that $\vec{P}$ is smooth enough in such a compact Brillouin zone. This is, of course, nothing but the Nielsen-Ninomiya theorem [54]. 


\section{Multiple and degenerate Weyl semimetal}

Problems of this kind have been dealt with in recent literature for a simple powerlike $\vec{P}$. The main prototype is

$$
\mathcal{D}=\left(\sigma^{+}\left(-i D_{+}\right)^{2}+\sigma^{-}\left(-i D_{-}\right)^{2}+\sigma^{3}\left(-i D_{3}\right)-D_{4}\right),
$$

where $D_{ \pm}=D_{1} \mp i D_{2}$ and $\sigma^{ \pm}=\left(\sigma^{1} \pm i \sigma_{2}\right) / 2$. This has been motivated by a merging of a pair of Dirac cones, initially separated in the Brillouin zone. A slight generalization of this can be achieved by elevating the power to an arbitrary positive $n$, and also replacing $-i D_{3}$ with

$$
P_{3}=C_{l}\left(-i D_{3}\right)^{l}+\cdots,
$$

where the ellipsis denotes lower-order monomials of $-i D_{3}$. As is clear from the general formalism, these subleading pieces are irrelevant for the problems at hand, so we may as well consider

$\mathcal{D}=\left(\sigma^{+}\left(-i D_{+}\right)^{n}+\sigma^{-}\left(-i D_{-}\right)^{n}+\sigma^{3} C_{l}\left(-i D_{3}\right)^{l}-D_{4}\right)$,

whose associated Dirac operator is

$$
\begin{aligned}
\mathcal{Q}=\left(\begin{array}{cc}
0 & \mathcal{D} \\
\mathcal{D}^{\dagger} & 0
\end{array}\right)= & \left(\gamma^{+}\left(-i D_{+}\right)^{n}+\gamma^{-}\left(-i D_{-}\right)^{n}\right. \\
& \left.+\gamma^{3} C_{l}\left(-i D_{3}\right)^{l}+\gamma^{4}\left(-i D_{4}\right)\right)
\end{aligned}
$$

with $\gamma^{ \pm}=\left(\gamma^{1} \pm i \gamma^{2}\right) / 2$. The anomaly computation for $n=2,3$ and $l=1$ with $C_{1}=1$ has been performed in recent literature [7,8]. Although this class of examples clearly falls under the general formalism above, we repeat the exercise in part as an concrete example of our general formulation, but also to show explicitly how the additional power $l$ enters the story.

The zeroth-order part of the squared Dirac operator is

$$
-\mathcal{Q}_{0}^{2}=\left(\left(\partial_{1}\right)^{2}+\left(\partial_{2}\right)^{2}\right)^{n}+C_{l}^{2}\left(\partial_{3}\right)^{2 l}+\left(\partial_{4}\right)^{2},
$$

so that, now with $X=(Z ; U, V)$ and $K=(p ; q, \tilde{q})$,

$$
\begin{aligned}
G_{s}^{(0)}(x+X ; x)= & \frac{1}{\sqrt{4 \pi s}} e^{-V^{2} / 4 s} \\
& \times \int \frac{d^{2} p d q}{(2 \pi)^{3}} e^{i(p \cdot Z+q U)} e^{-s\left(p_{1}^{2}+p_{2}^{2}\right)^{n}-s C_{l}^{2} q^{2 l}} .
\end{aligned}
$$

Again, the key quantity to compute is the second piece in

$$
\mathcal{Q}^{2}=\mathcal{P}_{\mu} \mathcal{P}_{\mu}+\frac{1}{4}\left[\gamma^{\mu}, \gamma^{\nu}\right]\left[\mathcal{P}_{\mu}, \mathcal{P}_{\nu}\right],
$$

and the relevant terms in $\delta \mathcal{Q}^{2}$ are

$$
\begin{aligned}
& \frac{1}{4}\left[\gamma^{\mu}, \gamma^{\nu}\right]\left[\mathcal{P}_{\mu}, \mathcal{P}_{\nu}\right]=\gamma^{1} \gamma^{2}\left(n^{2} F_{12}\right)\left(p_{+} p_{-}\right)^{n-1}+\cdots \\
& \quad+\gamma^{ \pm} \gamma^{3}\left(-n l\left(F_{13} \mp i F_{23}\right)\right) C_{l} q^{l-1}\left(p_{+}\right)^{n-1}+\cdots \\
& \quad+\gamma^{ \pm} \gamma^{4}\left(-n\left(F_{14} \mp i F_{24}\right)\right)\left(p_{+}\right)^{n-1}+\cdots \\
& \quad+\gamma^{3} \gamma^{4} F_{34} l C_{l} q^{l-1}+\cdots
\end{aligned}
$$

As before, the momentum accumulates through each iteration, and the relevant part of the heat kernel can be found at the second order of iteration,

$$
\begin{aligned}
& G_{s}(x+X ; x) \\
& =\cdots-\Gamma \frac{s^{3 / 2}}{\sqrt{4 \pi}}\left(l \times f(F(x)) e^{-V^{2} / 4 s}\right. \\
& \quad \times \int \frac{d^{2} p d q}{(2 \pi)^{3}}\left(p_{+} p_{-}\right)^{n-1} q^{l-1} e^{i p \cdot Z+i q U} e^{-s\left(\left(p^{2}\right)^{n}+C_{l}^{2}\left(q^{2}\right)^{l}\right)}+\cdots
\end{aligned}
$$

with

$$
f(F)=n^{2}\left(F_{12} F_{34}+F_{31} F_{24}+F_{23} F_{14}\right) .
$$

For even $l$, the $q$ integral vanishes, since the integrand is odd under $q \rightarrow-q$. For odd $l$, on the other hand, the momentum integral gives in the coincident limit

$$
\begin{aligned}
& \int \frac{d^{2} p}{(2 \pi)^{2}}\left(p^{2}\right)^{n-1} e^{-s\left(p^{2}\right)^{n}} \times \int \frac{d q}{2 \pi} C_{l} q^{l-1} e^{-s C_{l}^{2}\left(q^{2}\right)^{l}} \\
& =\frac{1}{4 \pi s} \frac{1}{n} \times \frac{1}{\sqrt{4 \pi s}} \frac{\operatorname{sgn}\left(C_{l}\right)}{l} .
\end{aligned}
$$

This brings us to

$$
N_{\vec{P}}=\left\{\begin{array}{ll}
\operatorname{sgn}\left(C_{l}\right) \cdot n & \text { for odd } l \\
0 & \text { for even } l
\end{array},\right.
$$

where $l$ does not add to the winding number but rather turns it on or off, depending on its value modulo 2 .

\section{DIAGRAMMATIC COMPUTATION OF THE ANOMALY}

One of the points that remains unresolved in the above anomaly computation via the Fujikawa viewpoint was the nature of the Wick rotation. Although the computation stands well defined as a generalized index problem for a spinor valued in a vector bundle over $\mathbb{R}^{d}$, its relation to quantum theories in the real world with the Lorentzian signature needs to be clarified further when the spatial momentum mixes in with the frequency. The usual Wick rotation $-i t \rightarrow \tau$ no longer works, because of such a mix, 
so it is necessary to readdress the issue from a Lorentzian viewpoint by computing the usual Feynman diagram and seeing how its result is connected to those above. In particular, this will give us a clearer picture of exactly what Wick rotation we have effectively performed, and also clarify why the general form of the anomaly remains intact, modulo a multiplicative constant, despite the higher inverse power of the momentum in the propagators.

For this, we consider the generalized fermion action $S=\int_{x} \mathcal{L}$, where

$$
\mathcal{L}=-\bar{\psi} \gamma^{\mu} \mathcal{P}_{\mu}(-i D) \psi+i M \bar{\psi} \psi
$$

It is a massive fermion of mass $M$, and the true object we need is a subtraction between an $M=0$ fermion and the Pauli-Villars fermion of $M$ in the $M \rightarrow \infty$ limit. Once we have this subtraction, the loop integral is finite, and we are free to perform a shift or change of the loop variables. When we perform such operations in the following, it is understood that we do the same simultaneously for both the $M=0$ fermion and the Pauli-Villars fermion contributions in the regularized integrand, so that it is justified.

We assume that $\mathcal{P}_{\mu}(-i D)$ is expandable in power series, and we show details for a particular order- $n$ term explicitly, and wherever we can replace the order- $n$ expression with the general $\mathcal{P}_{\mu}(k)$, we will do so. The action with an order- $n$ term is

$$
\begin{aligned}
& -\bar{\psi} \gamma^{\mu} \mathcal{P}_{\mu}(-i D) \psi+i M \bar{\psi} \psi \\
& \quad=-C_{\mu}^{\alpha_{1} \alpha_{2} \cdots \alpha_{n}} \bar{\psi} \gamma^{\mu}(-i D)_{\alpha_{1}}(-i D)_{\alpha_{2}} \cdots(-i D)_{\alpha_{n}} \psi+i M \bar{\psi} \psi .
\end{aligned}
$$

Either by the Noether method or by introducing the auxiliary chiral gauge field $A_{5}$ in $D=\partial+A+A_{5} \gamma^{5}$ and differentiating the action with respect to $A_{5}$, the chiral current is obtained as

$$
\begin{aligned}
j_{A}^{\mu}= & \sum_{s=1}^{n} C_{\nu}^{\alpha_{1} \cdots \alpha_{s-1} \mu \alpha_{s+1} \cdots \alpha_{n}}\left((i D)_{\alpha_{s-1}} \cdots\right. \\
& \left.\times(i D)_{\alpha_{1}} \bar{\psi}\right) \gamma^{\nu} \gamma^{5}(-i D)_{\alpha_{s+1}} \cdots(-i D)_{\alpha_{n}} \psi .
\end{aligned}
$$

Note that $D \psi=(\partial+A) \psi$ and $D \bar{\psi}=(\partial-A) \bar{\psi}$. Although we do not use the conserved vector current $j^{\mu}$, it has the same form as the above except for $\gamma^{5}$. Using the classical equation of motion, it is easily seen that

$$
\partial_{\mu} j_{A}^{\mu}=2 M \bar{\psi} \gamma^{5} \psi
$$

If there were no UV divergence in the correlation functions involving $j_{A}^{\mu}$, Eq. (4.4) would imply a Ward identity for the correlation functions,

$$
\partial_{\mu}\left\langle j_{A}^{\mu} \cdots\right\rangle=2 M\left\langle\bar{\psi} \gamma^{5} \psi \cdots\right\rangle
$$

which could be seen diagrammatically order by order in the loop expansion. We have checked this explicitly at one loop up to second order in the background gauge fields, which is relevant to the chiral anomaly. In showing this, one needs to shift or change the loop momenta, which is valid only when the integrand is UV finite. For a divergent diagram, such as the one we need to compute for the chiral anomaly, these operations are allowed only after subtracting the regularization contribution to make it finite-in our case, the PauliVillars fermion. The regularized chiral current is therefore

$$
j_{A, \text { reg }}^{\mu}=j_{A, M=0}^{\mu}-j_{A, M}^{\mu},
$$

which has now the valid Ward identity

$$
\partial_{\mu}\left\langle j_{A, \text { reg }}^{\mu} \cdots\right\rangle=-2 M\left\langle\bar{\psi}_{M} \gamma^{5} \psi_{M} \cdots\right\rangle,
$$

where $\psi_{M}$ is the Pauli-Villars fermion. The right-hand side of the above will be seen to be finite in the one-loop order that is sufficient to derive our chiral anomaly, ${ }^{5}$ and the chiral anomaly, whatever it is, is obtained by

$$
\mathcal{A}=\lim _{M \rightarrow \infty}-2 M\left\langle\bar{\psi}_{M} \gamma^{5} \psi_{M}\right\rangle .
$$

There is another viewpoint that leads to the same formula for the chiral anomaly. Consider a physical massive fermion of mass $M$. Its chiral current has the Ward identity

$$
\partial_{\mu} j_{A}^{\mu}=2 M \bar{\psi} \gamma^{5} \psi+\mathcal{A} .
$$

As $M \rightarrow \infty$, the fermion should decouple from the lowenergy regime, and the symmetry current and its Ward identity should be provided and saturated by other lowenergy degrees of freedom in the $M \rightarrow \infty$ limit. This means that the right-hand side of the above must vanish in the $M \rightarrow \infty$ limit.

In the diagrammatic evaluation of Eq. (4.8) up to second order in the background gauge fields, there are three diagrams to compute, as shown in Fig. 1. Since a nonvanishing $\gamma^{5}$ trace requires at least four $\gamma$ matrices, and each vertex and propagator contains at most one $\gamma$ matrix, the diagrams in Figs. 1(b) and 1(c) vanish trivially, and we only consider the triangle diagram in Fig. 1(a). The propagator in momentum space as a spinor matrix is ${ }^{6}$

\footnotetext{
${ }^{5}$ If the right-hand side remains divergent, although the degree of divergence should be reduced, one needs to introduce the second pair of Pauli-Villars fields to subtract such divergence. One continues to introduce the necessary Pauli-Villars fields until it becomes finite.

${ }^{6}$ We denote the 4-momentum in the Lorentzian signature by $k$, to be distinguished from the Euclidean 4-momentum $K$ or from the spatial 3-momentum $\vec{k}$. The precise relation between the Euclidean $K$ we used in Sec. II and the Lorentzian $k$ is more subtle than for the usual Dirac fermions. See the last part of this section.
} 


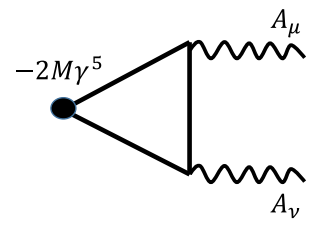

(a)

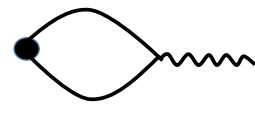

(b)

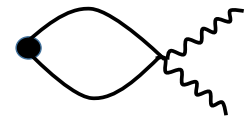

(c)

FIG. 1. The Feynman diagrams for $-2 M\left\langle\bar{\psi}_{M} \gamma^{5} \psi_{M}\right\rangle$.

$$
\left\langle\psi(k) \bar{\psi}\left(k^{\prime}\right)\right\rangle=(2 \pi)^{4} \delta^{(4)}\left(k+k^{\prime}\right) \frac{(-i)}{\gamma^{\mu} \mathcal{P}_{\mu}(k)-i M} .
$$

We need the interaction vertex only up to first order in the gauge field:

$$
\begin{aligned}
i S \sim & -\sum_{s=1}^{n} C_{\mu}^{\alpha_{1} \cdots \alpha_{n}}\left((i \partial)_{\alpha_{s-1}} \cdots(i \partial)_{\alpha_{1}} \bar{\psi}\right) \gamma^{\mu} \\
& \times A_{\alpha_{s}}(-i \partial)_{\alpha_{s+1}} \cdots(-i \partial)_{\alpha_{n}} \psi .
\end{aligned}
$$

The Feynman rule for this vertex, as shown in Fig. 2, is given by

$$
\begin{aligned}
& -\sum_{s=1}^{n} C_{\mu}^{\alpha_{1} \cdots \alpha_{n}} k_{\alpha_{1}} \cdots k_{\alpha_{s-1}}(k-q)_{\alpha_{s+1}} \cdots(k-q)_{\alpha_{n}} \gamma^{\mu} A_{\alpha_{s}}(q) \\
& \quad \equiv \Gamma(k, q) \cdot A(q)
\end{aligned}
$$

where $\psi$ carries a momentum $k-q, \bar{\psi}$ carries a momentum $-k$, and the momentum of the gauge field $A$ is $q$. Note that

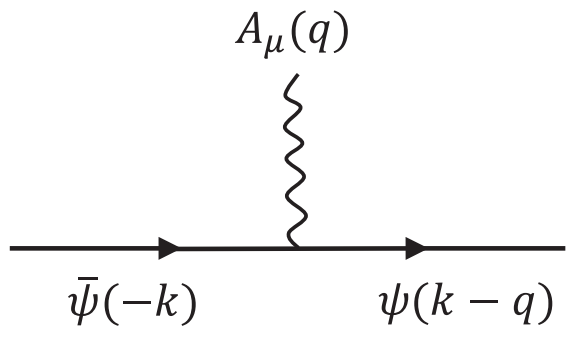

FIG. 2. The Feynman diagram for the interaction vertex.

$$
\Gamma(k, 0) \cdot A(q)=-\gamma^{\mu} \frac{\partial \mathcal{P}_{\mu}(k)}{\partial k_{\nu}} A_{\nu}(q)
$$

which will be used later.

It is easy to write the expression $-2 M\left\langle\bar{\psi}_{M} \gamma^{5} \psi_{M}\right\rangle$ of momentum $p$ as (where $\psi$ carries a momentum $k$ and $\bar{\psi}$ carries a momentum $p-k$ )

$$
\begin{aligned}
& 2 M \int_{q} \int_{k} \operatorname{Tr}\left(\gamma^{5} \frac{(-i)}{\gamma \cdot \mathcal{P}(k)-i M} \Gamma(k, q)\right. \\
& \cdot A(q) \frac{(-i)}{\gamma \cdot \mathcal{P}(k-q)-i M} \times \Gamma(k-q, p-q) \\
& \left.\quad \cdot A(p-q) \frac{(-i)}{\gamma \cdot \mathcal{P}(k-p)-i M}\right),
\end{aligned}
$$

where $\int_{k}=\int \frac{d^{4} k}{(2 \pi)^{4}}$ is the loop integration. This is equal to

$$
2 i M \int_{q} \int_{k} \frac{\operatorname{Tr}(\mathcal{I})}{\left(\mathcal{P}(k)^{2}+M^{2}-i \epsilon\right)\left(\mathcal{P}(k-q)^{2}+M^{2}-i \epsilon\right)\left(\mathcal{P}(k-p)^{2}+M^{2}-i \epsilon\right)},
$$

where $\mathcal{P}^{2}=\mathcal{P}_{\mu} \mathcal{P}^{\mu}$, and

$$
\operatorname{Tr}(\mathcal{I})=\operatorname{Tr}\left(\gamma^{5}(\gamma \cdot \mathcal{P}(k)+i M) \Gamma(k, q) \cdot A(q)(\gamma \cdot \mathcal{P}(k-q)+i M) \times \Gamma(k-q, p-q) \cdot A(p-q)(\gamma \cdot \mathcal{P}(k-p)+i M)\right) .
$$

Note that we introduce $-i \epsilon$ for the time-ordered correlation functions.

The nonzero $\gamma^{5}$ trace in the above requires precisely four $\gamma$ matrices, and

$$
\operatorname{Tr}(\mathcal{I})=i M\left(\operatorname{Tr}\left(\mathcal{I}_{1}\right)+\operatorname{Tr}\left(\mathcal{I}_{2}\right)+\operatorname{Tr}\left(\mathcal{I}_{3}\right)\right)
$$

where

$$
\begin{aligned}
& \operatorname{Tr}\left(\mathcal{I}_{1}\right)=\operatorname{Tr}\left(\gamma^{5}(\Gamma(k, q) \cdot A(q))(\gamma \cdot \mathcal{P}(k-q))(\Gamma(k-q, p-q) \cdot A(p-q))(\gamma \cdot \mathcal{P}(k-p))\right), \\
& \operatorname{Tr}\left(\mathcal{I}_{2}\right)=\operatorname{Tr}\left(\gamma^{5}(\gamma \cdot \mathcal{P}(k))(\Gamma(k, q) \cdot A(q))(\Gamma(k-q, p-q) \cdot A(p-q))(\gamma \cdot \mathcal{P}(k-p))\right), \\
& \operatorname{Tr}\left(\mathcal{I}_{3}\right)=\operatorname{Tr}\left(\gamma^{5}(\gamma \cdot \mathcal{P}(k))(\Gamma(k, q) \cdot A(q))(\gamma \cdot \mathcal{P}(k-q))(\Gamma(k-q, p-q) \cdot A(p-q))\right) .
\end{aligned}
$$

Since the $\gamma^{5}$ trace is totally antisymmetric with respect to four $\gamma$ matrices in each $\mathcal{I}_{1,2,3}$, we can shuffle the four factors inside each $\mathcal{I}_{1,2,3}$ up to sign changes. For the same reason, we can add any multiple of one factor to the other factor without changing the result. Adding $\operatorname{Tr}\left(\mathcal{I}_{2}\right)$ and $\operatorname{Tr}\left(\mathcal{I}_{3}\right)$, we have 


$$
\begin{aligned}
\operatorname{Tr}\left(\mathcal{I}_{2}\right) & +\operatorname{Tr}\left(\mathcal{I}_{3}\right) \\
= & \operatorname{Tr}\left(\gamma^{5}(\gamma \cdot \mathcal{P}(k))(\Gamma(k, q) \cdot A(q))\right. \\
& \times(\Gamma(k-q, p-q) \cdot A(p-q)) \\
& \times(\gamma \cdot \mathcal{P}(k-p)-\gamma \cdot \mathcal{P}(k-q))) .
\end{aligned}
$$

Subtracting the second factor from the last factor in $\operatorname{Tr}\left(\mathcal{I}_{1}\right)$,

$$
\begin{aligned}
\operatorname{Tr}\left(\mathcal{I}_{1}\right)= & \operatorname{Tr}\left(\gamma^{5}(\Gamma(k, q) \cdot A(q))(\gamma \cdot \mathcal{P}(k-q))\right. \\
& \times(\Gamma(k-q, p-q) \cdot A(p-q)) \\
& \times(\gamma \cdot \mathcal{P}(k-p)-\gamma \cdot \mathcal{P}(k-q))) .
\end{aligned}
$$

Adding these, we have

$$
\begin{aligned}
\operatorname{Tr}\left(\mathcal{I}_{1}\right) & +\operatorname{Tr}\left(\mathcal{I}_{2}\right)+\operatorname{Tr}\left(\mathcal{I}_{3}\right) \\
= & \operatorname{Tr}\left(\gamma^{5}(\gamma \cdot \mathcal{P}(k)-\gamma \cdot \mathcal{P}(k-q))\right. \\
& \times(\gamma \cdot \mathcal{P}(k-p)-\gamma \cdot \mathcal{P}(k-q)) \\
& \times(\Gamma(k, q) \cdot A(q))(\Gamma(k-q, p-q) \cdot A(p-q))) .
\end{aligned}
$$

The first factor vanishes linearly in the small- $q$ limit,

$$
\gamma \cdot \mathcal{P}(k)-\gamma \cdot \mathcal{P}(k-q) \approx \gamma^{\mu} \frac{\partial \mathcal{P}_{\mu}(k)}{\partial k_{\nu}} q_{\nu}+\cdots,
$$

which, together with $A(q)$, gives a first-order derivative of $A$ in coordinate space. The same is true for the second factor,

$\gamma \cdot \mathcal{P}(k-p)-\gamma \cdot \mathcal{P}(k-q) \approx-\gamma^{\mu} \frac{\partial \mathcal{P}_{\mu}(k)}{\partial k_{\nu}}(p-q)_{\nu}+\cdots$,

which combines with $A(p-q)$ to give another derivative of $A$ in coordinate space. If we truncate higher-order derivatives of $A$ in coordinate space, we need to set $q$ and $(p-q)$ in the other factors to zero. ${ }^{7}$ Then, the third and fourth factors become

$$
\Gamma(k, q) \cdot A(q) \rightarrow \Gamma(k, 0) \cdot A(q)=-\gamma^{\mu} \frac{\partial \mathcal{P}_{\mu}(k)}{\partial k_{\nu}} A_{\nu}(q),
$$

and

\footnotetext{
${ }^{7}$ We can make it less ad hoc by the observation that the expansion parameter is either $q / k$ or $(p-q) / k$. In the $M \rightarrow \infty$ limit, our final result of the chiral anomaly, which is finite, is dominated by the integration region of $k \sim M$, and the higherderivative terms are suppressed by additional powers of $\partial / M$, which vanish in $M \rightarrow \infty$.
}

$$
\Gamma(k-q, p-q) \cdot A(p-q) \rightarrow-\gamma^{\mu} \frac{\partial \mathcal{P}_{\mu}(k)}{\partial k_{\nu}} A_{\nu}(p-q) .
$$

Computing the trace, we obtain

$$
\begin{aligned}
\operatorname{Tr}(\mathcal{I})= & 4 M \operatorname{det}\left(\frac{\partial \mathcal{P}_{\mu}}{\partial k_{\nu}}\right) \epsilon^{\alpha \beta \alpha^{\prime} \beta^{\prime}}\left(i q_{\alpha}\right) \\
& \times A_{\beta}(q)\left(i(p-q)_{\alpha^{\prime}}\right) A_{\beta^{\prime}}(p-q),
\end{aligned}
$$

and in the remaining loop integral of Eq. (4.15) in the $M \rightarrow \infty$ limit, we can neglect $q$ and $p$ in the denominator, since it is dominated by $k \sim M \gg p, q$ region, and we arrive at the result for the chiral anomaly,

$$
\begin{gathered}
8 i M^{2} \int_{q} \epsilon^{\alpha \beta \alpha^{\prime} \beta^{\prime}}\left(i q_{\alpha}\right) A_{\beta}(q)\left(i(p-q)_{\alpha^{\prime}}\right) A_{\beta^{\prime}}(p-q) \\
\times \int_{k} \operatorname{det}\left(\frac{\partial \mathcal{P}_{\mu}(k)}{\partial k_{\nu}}\right) \frac{1}{\left(\mathcal{P}(k)^{2}+M^{2}-i \epsilon\right)^{3}},
\end{gathered}
$$

which is in coordinate space,

$$
\mathcal{A}=\epsilon^{\alpha \beta \alpha^{\prime} \beta^{\prime}} F_{\alpha \beta} F_{\alpha^{\prime} \beta^{\prime}} \int_{k} \operatorname{det}\left(\frac{\partial \mathcal{P}_{\mu}(k)}{\partial k_{\nu}}\right) \frac{2 i M^{2}}{\left(\mathcal{P}(k)^{2}+M^{2}-i \epsilon\right)^{3}} .
$$

The above $k$ integration can be performed in $\mathcal{P}$ space up to the winding number $N_{\mathcal{P}}$ explained before:

$$
\begin{gathered}
\int_{k} \operatorname{det}\left(\frac{\partial \mathcal{P}_{\mu}(k)}{\partial k_{\nu}}\right) \frac{2 i M^{2}}{\left(\mathcal{P}(k)^{2}+M^{2}-i \epsilon\right)^{3}} \\
=N_{\mathcal{P}} \int \frac{d^{4} \mathcal{P}}{(2 \pi)^{4}} \frac{2 i M^{2}}{\left(\mathcal{P}^{2}+M^{2}-i \epsilon\right)^{3}},
\end{gathered}
$$

and the Wick rotation $\mathcal{P}^{0} \rightarrow i \mathcal{P}_{E}^{0}$ gives

$$
\begin{aligned}
\int \frac{d^{4} \mathcal{P}}{(2 \pi)^{4}} \frac{2 i M^{2}}{\left(\mathcal{P}^{2}+M^{2}-i \epsilon\right)^{3}} & =-\int \frac{d^{4} \mathcal{P}_{E}}{(2 \pi)^{4}} \frac{2 M^{2}}{\left(\mathcal{P}_{E}^{2}+M^{2}\right)^{3}} \\
& =-\frac{1}{16 \pi^{2}},
\end{aligned}
$$

which finally gives the chiral anomaly

$$
\mathcal{A}=-N_{\mathcal{P}} \frac{1}{16 \pi^{2}} \epsilon^{\alpha \beta \alpha^{\prime} \beta^{\prime}} F_{\alpha \beta} F_{\alpha^{\prime} \beta^{\prime}} .
$$

This result is the same as Eq. (2.22) except for an overall factor of 2, which has a well-known origin: Here we are computing the anomaly of a single four-component Diraclike fermion, while in Secs. II and III we computed for twocomponent chiral fermions. Note that, again, the winding number $N_{\mathcal{P}}$ factors out cleanly; the final form of the integral 
is expressed in terms of $\mathcal{P}$ in the place of $K$, such that the only surviving information about $\mathcal{P}$ is how many times $\mathcal{P}$ covers the momentum space $\mathbb{R}^{4}$.

Specializing to the case of a nonrelativistic Weyl semimetal, where

$$
\mathcal{P}_{0}(k)=k_{0}+\Delta(\vec{k}), \quad \mathcal{P}_{a}(k)=P_{a}(\vec{k}), \quad a=1,2,3,
$$

we have

$$
\begin{aligned}
\int_{k} \operatorname{det} & \left(\frac{\partial \mathcal{P}_{\mu}(k)}{\partial k_{\nu}}\right) \frac{2 i M^{2}}{\left(\mathcal{P}(k)^{2}+M^{2}-i \epsilon\right)^{3}} \\
= & \int \frac{d^{3} \vec{k}}{(2 \pi)^{3}} \operatorname{det}\left(\frac{\partial P_{a}(\vec{k})}{\partial k_{b}}\right) \\
& \times \int \frac{d k^{0}}{(2 \pi)} \frac{2 i M^{2}}{\left(-\left(k^{0}-\Delta(\vec{k})\right)^{2}+\vec{P}(\vec{k})^{2}+M^{2}-i \epsilon\right)^{3}} .
\end{aligned}
$$

Note that $\Delta(\vec{k})$ can be simply removed by a shift of $k^{0}$ integration, and the chiral anomaly is not affected by $\Delta(\vec{k})$. Since $\Delta(\vec{k})$ determines the shape of the Fermi surface by $|\vec{P}(\vec{k})|=-\Delta(\vec{k}),{ }^{8}$ the chiral anomaly is independent of the occupation number of states, but is determined only by the topology of the level crossing point.

Performing the Wick rotation of $k^{0}$ after shifting to remove $\Delta(\vec{k})$, the $k_{E}^{0}$ integration is easily done to give

$$
-\frac{3}{8} M^{2} \int \frac{d^{3} \vec{k}}{(2 \pi)^{3}} \operatorname{det}\left(\frac{\partial P_{a}(\vec{k})}{\partial k_{b}}\right) \frac{1}{\left(\vec{P}(\vec{k})^{2}+M^{2}\right)^{5 / 2}} \text {. }
$$

Again, up to the winding number $N_{\vec{P}}$ of the map $\vec{k} \rightarrow \vec{P}(\vec{k})$, the above integral can be performed in $\vec{P}$ space:

$$
-N_{\vec{P}} \frac{3}{8} M^{2} \int \frac{d^{3} \vec{P}}{(2 \pi)^{3}} \frac{1}{\left(\vec{P}^{2}+M^{2}\right)^{5 / 2}}=-N_{\vec{p}} \frac{1}{16 \pi^{2}},
$$

which reproduces the previous result by the index theorem.

One important point of this computation is the Wick rotation, $\mathcal{P}^{0} \rightarrow i \mathcal{P}_{E}^{0}$, which is not the same as the Wick rotation of the Lorentzian time to the Euclidean time. In the presence of $\Delta(\vec{k})$, the latter cannot be justified, as the rotation of the contour $k_{0} \rightarrow i k_{4}$ can encounter a pole along the way. On the other hand, as far as the diagrammatic computation goes, the Wick rotation is merely a trick that allows efficient computation. Therefore, a different contour choice in the complex plane of $k_{0}$ such that $\mathcal{P}^{0} \rightarrow i \mathcal{P}_{E}^{0}$

\footnotetext{
${ }^{8}$ The Hamiltonian for the right-handed Weyl component is $\vec{P}(\vec{k}) \cdot \vec{\sigma}+\Delta(\vec{k})$.
}

occurs is perfectly acceptable, as long as it is consistent with the idea of the Feynman propagator.

\section{GENERALIZED SPINORS IN ODD DIMENSIONS}

In odd spacetime dimensions, neither the usual $\mathbb{Z}$-valued index nor the chiral anomaly exists. Instead, one finds discrete anomalies, which have been the focus of active investigations recently in the context of the topological insulators. In all such investigations, the main physical quantity of interest is the phase of the partition function. When the fermion is massless, the reality of the action naively implies that the partition function is real, yet the actual path integral generically produces a phase factor. And, much as in the anomaly computation in even dimensions, this becomes apparent under the inevitable regularization of the path integral.

This phase has been computed in Ref. [55] and shown to be proportional to the eta invariant,

$$
\eta \equiv \lim _{s \rightarrow 0} \sum \operatorname{sgn}(\lambda)|\lambda|^{-s},
$$

with the sum over the eigenvalues of the Dirac operator, which measures some notion of the asymmetry of the eigenvalues of the Dirac operators under a sign flip. In the physics community, this is sometimes misrepresented by its cousin, namely the Chern-Simons action:

$$
\left.\frac{\pi}{2} \eta\right|_{-i \sigma^{a}\left(\partial_{a}+A_{a}\right)}=\frac{1}{2} S_{\mathrm{CS}}(A)+\cdots .
$$

The ellipsis denotes certain nonlocal terms, which makes the entire expression gauge invariant even if the ChernSimons coefficient is not properly quantized: this has to be, since the eigenvalues that define the eta invariant are always gauge invariant no matter what [55].

In this section, we will extend our investigation using the modified heat kernel to Dirac fermions in odd spacetime dimensions, with high-derivative Dirac operators as before. We will explicitly work out a $d=3$ Dirac fermion with the higher-derivative action

$$
S_{3}=-\int d^{3} x \bar{\psi} \mathcal{Q}_{3} \psi
$$

for which the relevant Dirac operator is

$$
\mathcal{Q}_{3}=\sigma^{a} P_{a}(-i \vec{D}),
$$

again with an arbitrary smooth function $P_{a}$.

We are interested in the purely imaginary piece of

$$
W(A)=-\log \left(\int[D \bar{\psi} D \psi] e^{-S_{3}}\right),
$$


which we will denote as $W^{\text {odd }}$. We will find that this phase, or equivalently, the eta invariant of this modified Dirac operator, is such that

$W^{\text {odd }}= \pm\left. i \frac{\pi}{2} \eta\right|_{\sigma^{a} P_{a}}=N_{\vec{P}} \cdot\left( \pm \frac{i}{2} S_{\mathrm{CS}}(A)+\cdots\right)$,

with the same winding number $N_{\vec{P}}$ of the map $P_{a}$, as in Eq. (3.7). The same notation $P_{a}$ as in Sec. III is used here, because eventually we will connect to the $d=4$ system, with the fourth direction treated as the normal to a $d=3$ boundary. For Majorana fermions, the discussions below carry over straightforwardly by multiplying the effective action by $1 / 2$.

\section{A. Eta invariant for generalized spinors}

As has been studied thoroughly for $\vec{P}=-i \vec{D}$ in Ref. [55], and also reviewed more recently in the context of topological insulators in Ref. [17], the path integral of such a fermion would lead to an effective action whose imaginary part is particularly simple to compute. We start with the Pauli-Villars regularized partition function

$$
\prod \frac{\lambda}{\lambda+i M},
$$

with eigenvalues $\lambda$ and the regulator mass $M \rightarrow \pm \infty$. The phases of individual pieces are $-i(\pi / 2) \operatorname{sgn}(M)(\lambda)$, leading to [55]

$$
W(A)^{\text {odd }}= \pm i \frac{\pi}{2} \eta(A),
$$

with Eq. (5.1).

Exactly the same reasoning applies even if we replace the usual first-order Dirac operator with $\mathcal{Q}_{3}=\sigma^{a} P_{a}(-i \vec{D})$ :

$$
W\left(A^{(R)}\right)_{\vec{P}}^{\text {odd }}= \pm i \frac{\pi}{2} \eta_{\vec{P}}\left(A^{(R)}\right),
$$

where we use the subscript $\vec{P}$ to remember that the Dirac operator is replaced by $\mathcal{Q}_{3}$. The eta invariant has the usual definition,

$$
\eta_{\vec{P}}\left(A^{(R)}\right)=\lim _{s \rightarrow 0} \sum \operatorname{sgn}\left(\lambda_{\mathcal{Q}_{3}}\right)\left|\lambda_{\mathcal{Q}_{3}}\right|^{-s},
$$

where the sum is now over all eigenvalues of the operator $\mathcal{Q}_{3}$. We labeled the gauge field by the representation $R$ of $\psi$ explicitly for the purpose of clarifying the normalization.

As noted already, the local part of this effective action for the usual $P_{a}=-i D_{a}$, on the other hand, is the well-known Chern-Simons action,

$$
\frac{\pi}{2} \eta_{\vec{P}=-i \vec{D}}\left(A^{(R)}\right)=t_{2}(R) \cdot \frac{1}{2} S_{\mathrm{CS}}(A)+\cdots,
$$

where $S_{\mathrm{CS}}(A)$ is the properly quantized Chern-Simons action, such that its half appears when we integrate out the complex unit charge $\psi$ under $U(1)$ or $\psi$ in the fundamental representation in $S U(N)$, for example. $t_{2}(R)$ is the quadratic invariant that keeps track of the gauge representation $R$. When $R$ is the adjoint representation, for example, it happens to be equal to twice the dual Coxeter number, $t_{2}(R=$ adjoint $)=2 h^{\vee}$.

Here, we wish to show that, with general $\vec{P}$, the above relations are modified simply as

$$
\frac{\pi}{2} \eta_{\vec{P}(-i \vec{D})}=N_{\vec{P}} \cdot t_{2}(R) \cdot \frac{1}{2} S_{\mathrm{CS}}(A)+\cdots,
$$

with the same winding number $N_{\vec{P}}$ that appears in the $d=4$ chiral anomaly. Upon introducing a Pauli-Villars regulator with $M$, again, we write the imaginary part of the effective action as

$$
\begin{aligned}
& W\left(A^{(R)}\right)_{\vec{P}}^{\text {odd }} \\
& \quad=\left.\frac{1}{2} \sum\left[\log \left(\lambda_{\mathcal{Q}_{3}}+i M\right)-\log \left(\lambda_{\mathcal{Q}_{3}}-i M\right)\right]\right|_{M \rightarrow \pm \infty} ^{\text {finite }} .
\end{aligned}
$$

The relation as in Eq. (5.11) comes from how this effective action varies as we vary the gauge field:

$$
\begin{aligned}
\delta W\left(A^{(R)}\right)_{\vec{P}}^{\text {odd }} & =\left.\frac{1}{2} \sum\left[\frac{\delta \lambda_{\mathcal{Q}_{3}}}{\lambda_{\mathcal{Q}_{3}}+i M}-\frac{\delta \lambda_{\mathcal{Q}_{3}}}{\lambda_{\mathcal{Q}_{3}}-i M}\right]\right|_{M \rightarrow \pm \infty} \\
& =-\left.i M \cdot \operatorname{Tr}\left[\frac{\delta \mathcal{Q}_{3}}{\left(\mathcal{Q}_{3}\right)^{2}+M^{2}}\right]\right|_{M \rightarrow \pm \infty} \\
& =-\left.i M \cdot \int_{0}^{\infty} d s \operatorname{Tr}\left[\delta \mathcal{Q}_{3} e^{-s\left(\left(\mathcal{Q}_{3}\right)^{2}+M^{2}\right)}\right]\right|_{M \rightarrow \pm \infty}
\end{aligned}
$$

Although the scaling of $s$ looks different from those we used in the anomaly computation, the content is no different because of the $e^{-s M^{2}}$ term in the integrand. The large- $M$ limit confines the $s$ integral effectively to a region of $s<1 / M^{2}$, so again the small-s expansion of the heat kernel becomes sufficient. And the computation again boils down to a heat kernel one in the coincident limit.

As such, in $d=3$, the first iteration suffices:

$$
\begin{aligned}
G_{s}^{(1)}(y ; x)= & \int_{0}^{s} d t \int d^{3} z G_{s-t}^{(0)}(y ; z) \\
& \times\left(\left(\mathcal{Q}_{3}\right)^{2}-\vec{P}(-i \vec{\partial})^{2}\right) G_{t}^{(0)}(z ; x),
\end{aligned}
$$

with

$$
\begin{aligned}
G_{s}^{(0)}(x+X ; x) & \equiv\left\langle x+X\left|e^{-s \vec{P}(-i \vec{\partial})^{2}}\right| x\right\rangle \\
& =\int \frac{d^{3} k}{(2 \pi)^{3}} e^{i \vec{k} \cdot \vec{X}} e^{-s \vec{P}(\vec{k})^{2}}
\end{aligned}
$$

as we can see explicitly below. 
With nontrivial $\vec{P}$, we find again in the momentum space an expansion around a generic point $x$ :

$$
\begin{aligned}
\delta \mathcal{Q}_{3} & =\sigma^{a} \tilde{\partial}^{b} P_{a}(\vec{k}) \delta A_{b}(x)+\cdots \\
\left(\mathcal{Q}_{3}\right)^{2} & =\vec{P}(\vec{k})^{2}+i \epsilon^{a b c} \sigma^{a} F_{f g}(x) \tilde{\partial}^{f} P_{b}(\vec{k}) \tilde{\partial}^{g} P_{c}(\vec{k})+\cdots
\end{aligned}
$$

where the ellipsis denotes again those terms that are suppressed by small- $s$, or equivalently large- $M$, scaling. Since $\delta \mathcal{Q}_{3}$ carries a single $\sigma^{a}$, the trace over the spinor requires another $\sigma^{a}$, which can be supplied by the field strength term in $\mathcal{Q}_{3}^{2}$ shown above.

The trace over the two-component spinor indices leaves behind

$$
\begin{aligned}
\delta_{A(x)} W\left(A^{(R)}\right)_{\vec{P}}^{\text {odd }} & =\left.i M \cdot \int_{0}^{\infty} d s s e^{-s M^{2}} \int \frac{d^{3} k}{(2 \pi)^{3}} e^{-s \vec{P}(\vec{k})^{2}} \operatorname{det}\left(\frac{\partial P_{f}(\vec{k})}{\partial k_{g}}\right) \operatorname{tr}_{R}\left(\epsilon^{a b c} \delta A_{a}(x) F_{b c}(x)\right)\right|_{M \rightarrow \pm \infty} \\
& =N_{\vec{P}} \cdot\left(\left.i M \cdot \int_{0}^{\infty} d s s e^{-s M^{2}} \int \frac{d^{3} P}{(2 \pi)^{3}} e^{-s \vec{P}^{2}} \cdot \operatorname{tr}_{R}\left(\epsilon^{a b c} \delta A_{a}(x) F_{b c}(x)\right)\right|_{M \rightarrow \pm \infty}\right)
\end{aligned}
$$

at a generic point $x$, with $\vec{k}$ integrals and an $s$ integral, and $N_{\vec{P}}$ is the same winding number we encountered in Sec. III.

Let us count the factor of $M$ to ensure one ends up with a finite quantity: the three $P$ integrations will generate $s^{-3 / 2}$, so the final $s$ integral will be of the form

$$
\begin{aligned}
M \cdot \int_{0}^{\infty} d s s^{-1 / 2} e^{-s M^{2}} & =\operatorname{sgn}(M) \cdot \int_{0}^{\infty} d \tilde{s} \tilde{s}^{-1 / 2} e^{-\tilde{s}} \\
& =\operatorname{sgn}(M) \cdot \sqrt{\pi} .
\end{aligned}
$$

$M$ is scaled out, leaving behind only its sign, and as in the previous anomaly computation, other terms in $\left(\mathcal{Q}_{3}\right)^{2}$ can at most contribute pieces that scale inversely with the large $M$.

This way, the quantity inside the large parentheses remains finite in the limit and produces the variation of the imaginary part of the effective action due to a single two-component spinor with $\vec{P}=-i \vec{D}$. For $\vec{P}=-i \vec{D}$, in fact, this is precisely how one shows the relation (5.11), by starting with the above and integrating over $\delta \vec{A}(x)$ back to the Chern-Simons action. Therefore, we obtain at the end of the computation

$W\left(A^{(R)}\right)_{\vec{P}}^{\text {odd }}=N_{\vec{P}} \cdot\left( \pm i t_{2}(R) \cdot \frac{1}{2} S_{\mathrm{CS}}(A)+\cdots\right)$

or

$$
\begin{aligned}
W\left(A^{(R)}\right)_{\vec{P}}^{\text {odd }} & =N_{\vec{P}} \cdot\left( \pm i \frac{\pi}{2} \eta_{\vec{P}=-i \vec{D}}\left(A^{(R)}\right)\right) \\
& =N_{\vec{P}} \cdot W\left(A^{(R)}\right)_{\vec{P}=-i \vec{D}}^{\text {odd }},
\end{aligned}
$$

given the general relation between the Chern-Simons action and the eta invariant in Eq. (5.11), and also from how the effective action has to be gauge invariant.

Generalization to the higher dimension $d=2 n-1$ is also straightforward. It is clear that the variation of the effective action (5.18) can be easily extended to $d=(2 n-1)$ and produce

$$
\delta_{A(x)} W_{\mathcal{Q}_{2 n-1}}^{\text {odd }} \sim N_{\vec{P}} \cdot \operatorname{tr}\left(\delta A \wedge F^{n-1}\right),
$$

now expressed as the $d$ form, for the generalized Dirac operator

$$
\mathcal{Q}_{2 n-1}=\gamma^{a} P_{a}(-\vec{D}),
$$

with $(2 n-1)$-dimensional Dirac matrices $\gamma^{a}$. On the other hand, the Chern-Simons density is defined via

$$
d S_{\mathrm{CS}}=\frac{1}{n !(2 \pi i)^{n}} \int \operatorname{tr} F^{n}
$$

so its variation is such that

$$
\begin{aligned}
d\left[\delta_{A(x)} S_{\mathrm{CS}}\right] & =\frac{1}{(n-1) !(2 \pi i)^{n}} \cdot \operatorname{tr}\left((d(\delta A)+A \delta A+\delta A A) F^{n-1}\right) \\
& =d\left[\frac{1}{(n-1) !(2 \pi i)^{n}} \cdot \operatorname{tr}\left(\left(\delta A(x) \wedge F^{n-1}(x)\right)\right]\right.
\end{aligned}
$$

which brings us back to

$$
\begin{aligned}
W\left(A^{(R)}\right)_{\vec{P}}^{\text {odd }} & =N_{\vec{P}} \cdot\left( \pm \frac{i}{2} S_{\mathrm{CS}}\left(A^{(R)}\right)+\cdots\right) \\
& =N_{\vec{P}} \cdot\left( \pm i \frac{\pi}{2} \eta_{\vec{P}=-i \vec{D}}\left(A^{(R)}\right)\right)
\end{aligned}
$$

following the same pattern we saw for $d=3$. 


\section{B. Boundary fermions and the APS-like index theorem}

When a $d$-dimensional manifold $\mathcal{M}_{d}$ has a boundary $\Sigma_{d-1}$, an Atiyah-Patodi-Singer (APS) index problem on $\mathcal{M}_{d}$ can be formulated via the extension of the manifold by attaching a semi-infinite cylinder with the cross section $\Sigma_{d-1}$. Imposing the square normalizability condition for the ground states, one finds

$$
\mathcal{I}_{\mathcal{M}_{d}}^{\mathrm{APS}}=\mathcal{I}_{\mathcal{M}_{d}}^{\mathrm{bulk}}-\frac{\eta_{\Sigma_{d-1}}}{2}
$$

where $\eta_{\Sigma_{d-1}}$ is the usual eta invariant of $\Sigma_{d-1}$.

Is there a way to extend this result to the generalized Dirac problem of our kind? Extending the covariant derivative to include the spin connection-say, $\nabla_{\mu}$-is no big deal. However, one also needs a covariantly constant tensor $C_{a}^{\mu_{1} \cdots \mu_{l}}$ such that operators such as

$$
\gamma^{a} C_{a}^{\mu_{1} \cdots \mu_{l}} \nabla_{\mu_{1}} \cdots \nabla_{\mu_{l}}
$$

can be used in place of the ordinary $\gamma^{a} e_{a}^{\mu} \nabla_{\mu}$ with the vielbein $e_{a}^{\mu}$. Such a tensor $C$ implies reduced holonomy, yet the latter is classified completely and known to be rather sparse. As such, a general higher-derivative Dirac operator with the curved geometry is generally difficult to construct.

When both $\mathcal{M}_{d}$ and $\Sigma_{d-1}$ are flat, one other hand, our discussions so far do imply an APS-like index theorem. The easiest is $\mathcal{M}_{d}=\mathbb{T}^{d-1} \times \mathbb{R}_{+}$with the boundary $\Sigma_{d-1}=$ $\mathbb{T}^{d-1}$ at the origin of $\mathbb{R}_{+}$. With the generalized Dirac operator as in Eq. (3.1),

$$
\mathcal{Q}_{d-1}-D_{d}=\gamma^{a} P_{a}(-i \vec{D})-D_{d}
$$

and thus

$$
\begin{aligned}
\mathcal{Q}_{d} & =\gamma^{a} P_{a}(-i \vec{D})+\gamma^{d}\left(-i D_{d}\right) \\
& =\left(\begin{array}{cc}
0 & \mathcal{Q}_{d-1}-D_{d} \\
\mathcal{Q}_{d-1}+D_{d} & 0
\end{array}\right),
\end{aligned}
$$

as in Eq. (3.3), with the direction $d$ considered as the normal to $\Sigma_{d-1}$. We have already seen that the bulk part of the index is

$$
\mathcal{I}_{\mathcal{Q}_{d}}^{\text {bulk }}=N_{\mathcal{P}} \cdot \mathcal{I}^{\text {bulk }}
$$

while the eta invariant is similarly enhanced as

$$
\frac{\eta_{\mathcal{Q}_{d-1}}}{2}=N_{\mathcal{P}} \cdot \frac{\eta}{2}
$$

With these, we can easily retrace the steps found in the Appendix of Ref. [55] with the modified heat kernel we have discussed so far, and arrive at a generalized APS index theorem:

$$
\begin{aligned}
\mathcal{I}_{\mathcal{Q}_{d}}= & N_{\vec{P}} \cdot\left(\frac { 1 } { n ! ( 2 \pi i ) ^ { n } } \int _ { \mathbb { T } ^ { 2 n - 1 } \times \mathbb { R } _ { + } } \operatorname { t r } \left(F^{(R)} \wedge \cdots\right.\right. \\
& \left.\left.\wedge F^{(R)}\right)-\frac{\eta^{\mathbb{T}^{2 n-1}}\left(A^{(R)}\right)}{2}\right)
\end{aligned}
$$

for any even dimensions $d=2 n$.

This form of the APS-like index theorem would suffice for understanding interfaces between topological insulators and ordinary insulators, by considering physics very near the boundary. As before, the factor $N_{\vec{P}}$ can be realized either as $N_{\vec{p}}$ many flavors of ordinary boundary fermions, or a single fermion with a higher-order Dirac operator with the winding number $N_{\vec{P}}$.

\section{ACKNOWLEDGMENTS}

We are grateful to Gil-Young Cho, Paolo Glorioso, Byungmin Kang, Dam Son, Misha Stephanov, and Raju Venugopalan for helpful discussions. H.-U. Y. appreciates the support and hospitality of KIAS during his visit when this work was initiated. The work of H.-U. Y. is supported by the U.S. Department of Energy, Office of Science, Office of Nuclear Physics, with Grant No. DEFG0201ER41195, and within the framework of the Beam Energy Scan Theory (BEST) Topical Collaboration. P. Y. is supported by a KIAS individual grant (No. GP005704) at the Korea Institute for Advanced Study.
[1] S. L. Adler, Axial vector vertex in spinor electrodynamics, Phys. Rev. 177, 2426 (1969).

[2] J. S. Bell and R. Jackiw, A PCAC puzzle: $\pi^{0} \rightarrow \gamma \gamma$ in the $\sigma$ model, Nuovo Cimento A 60, 47 (1969).

[3] K. Fujikawa, Path Integral Measure for Gauge Invariant Fermion Theories, Phys. Rev. Lett. 42, 1195 (1979).
[4] X. Wan, A. M. Turner, A. Vishwanath, and S. Y. Savrasov, Topological semimetal and Fermi-arc surface states in the electronic structure of pyrochlore iridates, Phys. Rev. B 83, 205101 (2011).

[5] A. A. Burkov and L. Balents, Weyl Semimetal in a Topological Insulator Multilayer, Phys. Rev. Lett. 107, 127205 (2011). 
[6] G. Xu, H. Weng, Z. Wang, X. Dai, and Z. Fang, Chern Semi-Metal and Quantized Anomalous Hall Effect in $\mathrm{HgCr}_{2} \mathrm{Se}_{4}$, Phys. Rev. Lett. 107, 186806 (2011).

[7] Z. M. Huang, J. Zhou, and S. Q. Shen, Topological responses from chiral anomaly in multi-Weyl semimetals, Phys. Rev. B 96, 085201 (2017).

[8] L. Lepori, M. Burrello, and E. Guadagnini, Axial anomaly in multi-Weyl and triple-point semimetals, J. High Energy Phys. 06 (2018) 110.

[9] E. Witten, Supersymmetry and Morse theory, J. Diff. Geom. 17, 661 (1982).

[10] D. J. Thouless, M. Kohmoto, M. P. Nightingale, and M. den Nijs, Quantized Hall Conductance in a Two-Dimensional Periodic Potential, Phys. Rev. Lett. 49, 405 (1982).

[11] M. C. Chang and Q. Niu, Berry phase, hyperorbits, and the Hofstadter spectrum: Semiclassical dynamics in magnetic Bloch bands, Phys. Rev. B 53, 7010 (1996).

[12] G. Sundaram and Q. Niu, Wave-packet dynamics in slowly perturbed crystals: Gradient corrections and Berry-phase effects, Phys. Rev. B 59, 14915 (1999).

[13] K. Yu. Bliokh, Topological spin transport of a relativistic electron, Europhys. Lett. 72, 7 (2005).

[14] L. Fu, C. L. Kane, and E. J. Mele, Topological Insulators in Three Dimensions, Phys. Rev. Lett. 98, 106803 (2007).

[15] J.E. Moore and L. Balents, Topological invariants of time-reversal-invariant band structures, Phys. Rev. B 75, 121306(R) (2007).

[16] X. L. Qi, T. L. Hughes, and S. C. Zhang, Topological field theory of time-reversal-invariant insulators, Phys. Rev. B 78, 195424 (2008).

[17] E. Witten, Fermion path integrals and topological phases, Rev. Mod. Phys. 88, 035001 (2016).

[18] D. Xiao, J. r. Shi, and Q. Niu, Berry Phase Correction to Electron Density of States in Solids, Phys. Rev. Lett. 95, 137204 (2005); Erratum, Phys. Rev. Lett. 95, 169903 (2005).

[19] D. T. Son and N. Yamamoto, Berry Curvature, Triangle Anomalies, and the Chiral Magnetic Effect in Fermi Liquids, Phys. Rev. Lett. 109, 181602 (2012).

[20] M. A. Stephanov and Y. Yin, Chiral Kinetic Theory, Phys. Rev. Lett. 109, 162001 (2012).

[21] J. W. Chen, S. Pu, Q. Wang, and X. N. Wang, Berry Curvature and Four-Dimensional Monopoles in the Relativistic Chiral Kinetic Equation, Phys. Rev. Lett. 110, 262301 (2013).

[22] C. Duval and P. A. Horvathy, Chiral fermions as classical massless spinning particles, Phys. Rev. D 91, 045013 (2015).

[23] N. Mueller and R. Venugopalan, Worldline construction of a covariant chiral kinetic theory, Phys. Rev. D 96, 016023 (2017).

[24] H. B. Nielsen and M. Ninomiya, Adler-Bell-Jackiw anomaly and Weyl fermions in crystal, Phys. Lett. 130B, 389 (1983).

[25] A. A. Zyuzin and A. A. Burkov, Topological response in Weyl semimetals and the chiral anomaly, Phys. Rev. B 86, 115133 (2012).

[26] P. Goswami and S. Tewari, Axionic field theory of $(3+1)-$ dimensional Weyl semimetals, Phys. Rev. B 88, 245107 (2013).
[27] G. Basar, D. E. Kharzeev, and H. U. Yee, Triangle anomaly in Weyl semimetals, Phys. Rev. B 89, 035142 (2014).

[28] K. Landsteiner, Anomalous transport of Weyl fermions in Weyl semimetals, Phys. Rev. B 89, 075124 (2014).

[29] J. Ma and D. A. Pesin, Dynamic Chiral Magnetic Effect and Faraday Rotation in Macroscopically Disordered Helical Metals, Phys. Rev. Lett. 118, 107401 (2017).

[30] D. E. Kharzeev, L. D. McLerran, and H. J. Warringa, The effects of topological charge change in heavy ion collisions: "Event-by-event $P$ and $C P$ violation", Nucl. Phys. A803, 227 (2008).

[31] D. E. Kharzeev, The chiral magnetic effect and anomalyinduced transport, Prog. Part. Nucl. Phys. 75, 133 (2014).

[32] K. Fukushima, D. E. Kharzeev, and H. J. Warringa, The chiral magnetic effect, Phys. Rev. D 78, 074033 (2008).

[33] A. Vilenkin, Equilibrium parity violating current in a magnetic field, Phys. Rev. D 22, 3080 (1980).

[34] D. Kharzeev and A. Zhitnitsky, Charge separation induced by $P$-odd bubbles in QCD matter, Nucl. Phys. A797, 67 (2007).

[35] J. Erdmenger, M. Haack, M. Kaminski, and A. Yarom, Fluid dynamics of $R$-charged black holes, J. High Energy Phys. 01 (2009) 055.

[36] N. Banerjee, J. Bhattacharya, S. Bhattacharyya, S. Dutta, R. Loganayagam, and P. Surowka, Hydrodynamics from charged black branes, J. High Energy Phys. 01 (2011) 094.

[37] T. Jungwirth, Q. Niu, and A. H. MacDonald, Anomalous Hall Effect in Ferromagnetic Semiconductors, Phys. Rev. Lett. 88, 207208 (2002).

[38] Z. Fang et al., Anomalous Hall effect and magnetic monopoles in momentum-space, Science 302, 92 (2003).

[39] Y. Yao, L. Kleinman, A. H. MacDonald, J. Sinova, T. Jungwirth, D.-s. Wang, E. Wang, and Q. Niu, First Principles Calculation of Anomalous Hall Conductivity in Ferromagnetic bcc Fe, Phys. Rev. Lett. 92, 037204 (2004).

[40] F. D. M. Haldane, Berry Curvature on the Fermi Surface: Anomalous Hall Effect as a Topological Fermi-Liquid Property, Phys. Rev. Lett. 93, 206602 (2004).

[41] Q. Li, D. E. Kharzeev, C. Zhang, Y. Huang, I. Pletikosić, A. V. Fedorov, R. D. Zhong, J. A. Schneeloch, G. D. Gu, and T. Valla, Observation of the chiral magnetic effect in $\mathrm{ZrTe}_{5}$, Nat. Phys. 12, 550 (2016).

[42] X. Huang et al., Observation of the Chiral-AnomalyInduced Negative Magnetoresistance in 3D Weyl Semimetal TaAs, Phys. Rev. X 5, 031023 (2015).

[43] C. Zhang et al., Signatures of the Adler-Bell-Jackiw chiral anomaly in a Weyl Fermion semimetal, Nat. Commun. 7, 0735 (2016).

[44] D. E. Kharzeev, J. Liao, S. A. Voloshin, and G. Wang, Chiral magnetic and vortical effects in high-energy nuclear collisions: Status report, Prog. Part. Nucl. Phys. 88, 1 (2016).

[45] V. Koch, S. Schlichting, V. Skokov, P. Sorensen, J. Thomas, S. Voloshin, G. Wang, and H. U. Yee, Status of the chiral magnetic effect and collisions of isobars, Chin. Phys. C 41, 072001 (2017).

[46] V. Dwivedi and M. Stone, Classical chiral kinetic theory and anomalies in even space-time dimensions, J. Phys. A 47, 025401 (2014).

[47] K. Fujikawa, Quantum anomaly and geometric phase: Their basic differences, Phys. Rev. D 73, 025017 (2006). 
[48] K. Fujikawa, Characteristics of chiral anomaly in view of various applications, Phys. Rev. D 97, 016018 (2018).

[49] N. Mueller and R. Venugopalan, Constructing phase space distributions with internal symmetries, Phys. Rev. D 99, 056003 (2019).

[50] H. P. McKean, Jr. and I. M. Singer, Curvature and the eigenvalues of the Laplacian, J. Diff. Geom. 1, 43 (1967).

[51] L. Alvarez-Gaume and E. Witten, Gravitational anomalies, Nucl. Phys. B234, 269 (1984).
[52] D. Ghim, C. Hwang, and P. Yi, Generalized Euler index, holonomy saddles, and wall-crossing, arXiv:1909.11092.

[53] T. T. Wu and C. N. Yang, Dirac monopole without strings: Monopole harmonics, Nucl. Phys. B107, 365 (1976).

[54] H. B. Nielsen and M. Ninomiya, No-go theorem for regularizing chiral fermions, Phys. Lett. 105B, 219 (1981).

[55] L. Alvarez-Gaume, S. Della Pietra, and G. W. Moore, Anomalies and odd dimensions, Ann. Phys. (N.Y.) 163, 288 (1985). 